Portland State University

PDXScholar

$10-29-1990$

\title{
A Comparison of Circadian Rhythms in Day and Night Shift Workers
}

Mary Lee Blood

Portland State University

Follow this and additional works at: https://pdxscholar.library.pdx.edu/open_access_etds

Part of the Physiology Commons, and the Psychology Commons

Let us know how access to this document benefits you.

Recommended Citation

Blood, Mary Lee, "A Comparison of Circadian Rhythms in Day and Night Shift Workers" (1990).

Dissertations and Theses. Paper 3991.

https://doi.org/10.15760/etd.5875

This Thesis is brought to you for free and open access. It has been accepted for inclusion in Dissertations and Theses by an authorized administrator of PDXScholar. Please contact us if we can make this document more accessible: pdxscholar@pdx.edu. 
AN ABSTRACT OF THE THESIS OF Mary Lee Blood for the Master of Science in Psychology presented October 29, 1990.

Title: A Comparison of Circadian Rhythms in Day and Night Shift Workers.

APPROVED BY THE MEMBERS OF THE THESIS COMMITTEE:

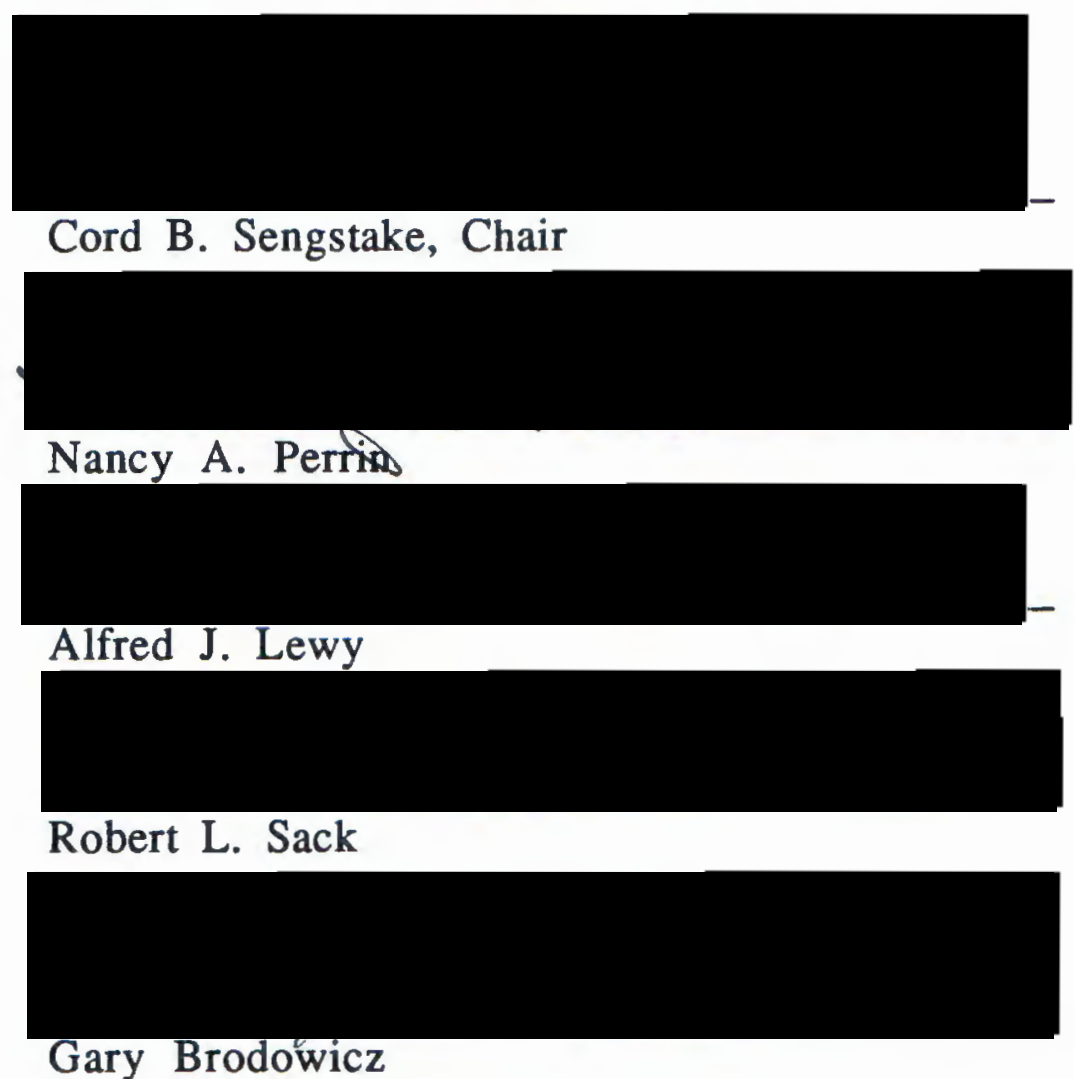

The present study examined whether and to what extent physiological rhythms of long-term night workers become adapted to their unconventionally scheduled hours of work and sleep and how 
the degree of adaptation would be reflected in ratings of sleep quality, mood, anxiety, energy and satisfaction.

Many physiological functions such as the release of hormones, excretion of metabolites and fluctuation of core temperature follow predictable circadian (24-hour) rhythms that are regulated by a pacemaker thought to be located in the suprachiasmatic nucleus $(\mathrm{SCN})$ of the hypothalamus. The timing of the production of the hormone melatonin was chosen as an indicator of physiological adaptation because its rhythm closely reflects the phase of the SCN. Ordinarily, melatonin is produced only at night and its onset, generally occurring one to several hours prior to sleep, can be determined with a high degree of accuracy. The melatonin rhythm is masked (obscured) only by bright light as opposed to other common measures which may be masked by sleep, activity, diet and stress. Therefore if bright light is avoided during the time of measurement, the melatonin rhythm provides an accurate reflection of its circadian pacemaker.

Nine night workers were studied at a local hospital following their last shift of the week. They entered dim light and $5 \mathrm{ml}$ blood samples were drawn hourly for the next 24-hours to determine the timing of their dim light melatonin onset (DLMO). A comparison group of nine day workers was also studied, however, samples were taken for only six hours surrounding the time of the expected DLMO (i.e, 1800 - 2300). Subjects kept daily diaries for the two weeks 
prior to admission, recording sleep times, sleep quality, sleepiness, mood, anxiety, energy and light exposure. Subjects also completed several scales in order to assess mood state, neuroticism, extraversion and diurnal (morning vs. evening) orientation for best functioning.

The timing of night workers' DLMOs (median $=14.47 \mathrm{~h}$ ) was remarkably different from that of day workers (median $=21.30 \mathrm{~h}$ ) $(p<.001$, Mann-Whitney $\mathrm{U}$ test), suggesting that for most night workers, a great deal of physiological adaptation had taken place. An unexpected finding was that the night workers' DLMOs occurred at a delayed phase in relation to sleep. Despite this, night workers obtained similar amounts of sleep and rated sleep quality comparably to day workers. Although night workers tended to sleep at more conventional times and obtain more total sleep on their non work days, they rated sleep quality, mood, anxiety and energy comparably to work days.

There was a great deal of variability in night workers' scores on psychological measures. However, for the most part, scores fell within the average range compared to normative samples. With the possible exception of neuroticism scores, which followed the expected pattern for all but two of the night workers, none of the other measures seemed promising as predictors of degree of adaptation.

These results suggest that this group of night workers is moderately well adapted to a schedule of nighttime work and 
daytime sleep. In most cases, lack of physiological adaptation is seen in an "overly delayed" circadian phase. Several clinical treatments are suggested as ways in which better adaptation might be obtained. Scheduling sleep so it occurs at a later time, more in phase with the circadian pacemaker is a simple suggestion, but perhaps the least agreeable to the night worker. Treatments of appropriately timed bright light and/or exogenous melatonin administration are discussed as possible techniques that may be utilized to advance the circadian pacemaker so that it is better synchronized with work and preferred sleep times. 


\title{
A COMPARISON OF CIRCADIAN RHYTHMS IN DAY AND NIGHT SHIFT WORKERS
}

by

MARY LEE BLOOD

A thesis submitted in partial fulfillment of the requirements for the degree of

\author{
MASTER OF SCIENCE \\ in \\ PSYCHOLOGY
}

Portland State University

1990 
TO THE OFFICE OF GRADUATE STUDIES:

The members of the Committee approve the thesis of Mary Lee Blood presented October 29, 1990.

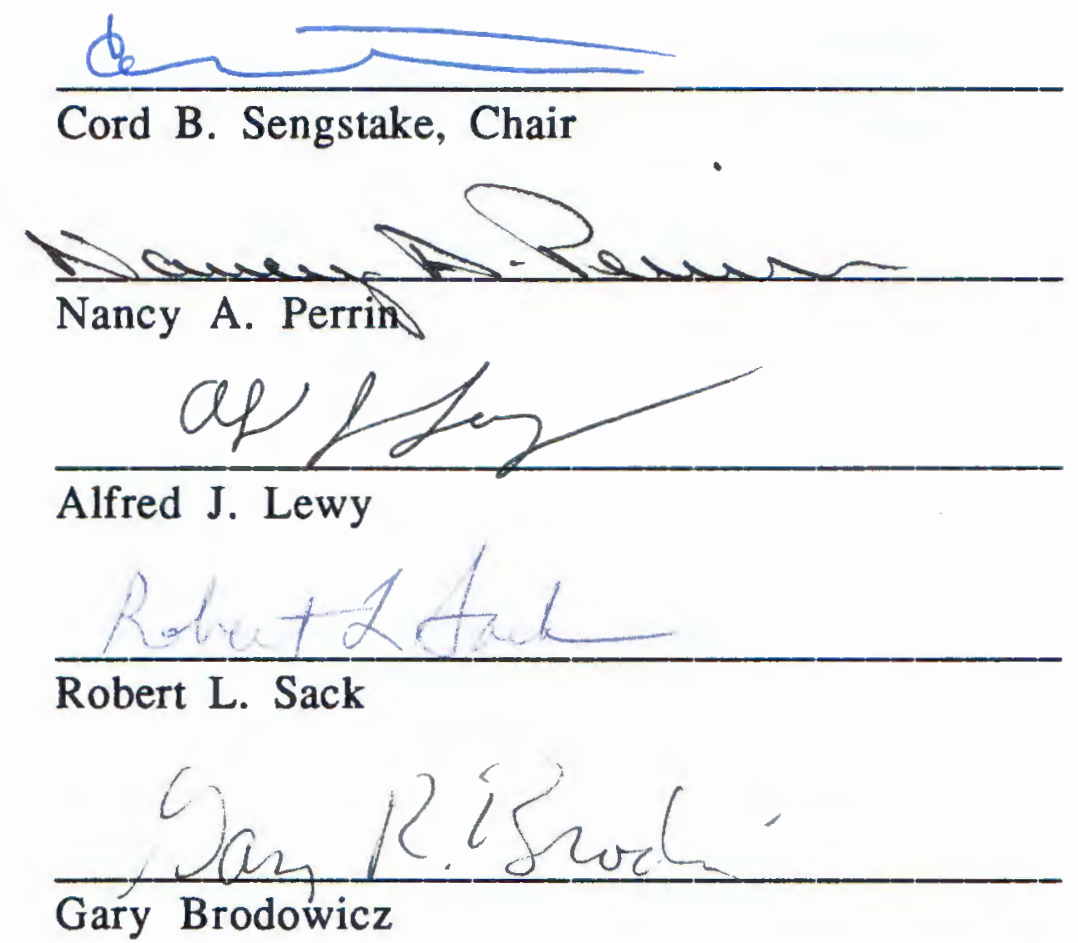

\section{APPROVED:}

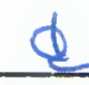

Cord B. Sengstake, Chair, Department of Psychology

\section{Willia stoven}

C. William Savery, Interim Vice frovost for Graduate Studies and Research 


\section{ACKNOWLEDGEMENTS}

I would like to thank the staff of the Sleep and Mood

Disorders Laboratory at Oregon Health Sciences University. Special Thanks to Greg Clarke, Mary Cardoza, Vance Bauer, Jaqueline Authier, David Wesche and Carol Simonton. I would also like to thank Dr. Robert Sack, Dr. Alfred Lewy and Dr. Jeanne Latham for their medical assistance. I wish also to thank the nursing and dietary staff of the Clinical Research Center. 


\section{TABLE OF CONTENTS}

\section{PAGE}

ACKNOWLEDGEMENTS iii LIST OF FIGURES .vii

\section{CHAPTER}

I INTRODUCTION .................................................................. 1

II RESEARCH ON THE CIRCADIAN RHYTHMS OF NIGHT WORKERS ................................ 6

Field Studies ............................................................. 6

Laboratory Studies ...................................................... 7

Masking and the Use of Constant Routines $\quad \ldots . .8$

Melatonin .................................................................... 12

Personality ............................................................. 14

III RATIONALE AND OUTLINE OF THE STUDY ............ 16

Physiological Adaptation ....................................... 16

Psychological Adaptation ...................................... 18

IV METHODOLOGY AND PROCEDURES .............................. 19

Subjects ....................................................................... 19 
Procedures

Night Workers

Day Workers

Procedures Common to Both Groups

Analysis of Physiological Samples

V RESULTS OF THE STUDY

27

Physiological and Behavioral Measures............ 27

Dim Light Melatonin Onsets

Sleep

Psychological Measures

34

Profile of Mood States

Eysenck Personality Inventory

Morningness Eveningness Questionnaire

General Health and Adjustment Questionnaire

Life Satisfaction

Self-reported Daily Ratings

VI CONCLUSIONS AND RECOMENDATIONS

41

REFERENCES 


\section{APPENDICES}

A DAILY DIARY AND INSTRUCTIONS ....................................6 60

B GRAPHIC REPRESENTATION OF INDIVIDUAL NIGHT WORKER SLEEP PATTERNS ..................................................... 65

C PHYSICAL SYMPTOMS ........................................................... 75

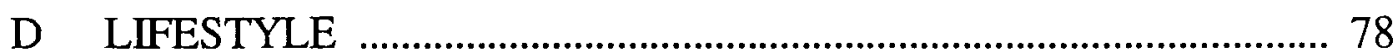

E CASE REPORTS FOR INDIVIDUAL NIGHT WORKERS ............. 81 


\section{LIST OF FIGURES}

1. The relationship of the dim light melatonin onset and average sleep periods in day and night shift workers

2. Night workers' melatonin onsets with proximal sleep and work periods.

3. Percentage of day and night shift workers asleep during an average 24 -hour period

4. Sleep strategies in two "moderately morning" type night workers 


\section{CHAPTER I}

\section{INTRODUCTION}

Shift work, which involves working irregular or nontraditional hours, is a part of working life for millions of Americans. Estimates on the percentage of the population who are exposed to work patterns that involve disruption of the normal daytime work/nighttime sleep orientation vary. According to the Bureau of Labor statistics, approximately 18 percent of the United States labor force worked in shift systems in 1977 (Johnson, Tepas, Colquhoun and Colligan, 1981). Czeisler (1982) estimates that $26.8 \%$ of workers in the United States labor force are exposed to work that requires major alterations in their normal day/night orientation. Analysis of data from The National Survey of Personal Health Practices and Consequences revealed that as of spring 1980, 18 percent of women in the US labor force and 26 percent of men worked variable (i.e., some combination of day and night) shifts (Gorden, Cleary, Parker and Czeisler, 1986).

Shift work is by no means unique to the United States. For example, $21.9 \%$ of the workers in France, $20.4 \%$ in the Netherlands, $23.3 \%$ in Japan and $18.2 \%$ in the United Kingdom are employed in shift work (Rutenfranz, Knauth and Colquhoun, 1976). Moore-Ede, Sulzman and Fuller (1982), estimate that as many as 60 million people in the industrialized nations participate in some form of shift 
work. It is not likely that shift work will be a passing phenomenon. In the United States some industries have over half of their employees on shift systems and the proportion of manufacturers operating on multiple shift systems has been increasing steadily by approximately $3 \%$ every year (Johnson et al., 1981).

There are many reasons why modern technological societies require that work be performed around the clock. The chemical, steel and energy industries involve technological processes that can not be interrupted. The high capital investment involved in some manufacturing industries requires that expensive machinery be operated around the clock in order to be cost-effective. There are also increased requirements for 24-hour medical, transportation and emergency services. As increasing numbers of individuals engage in nontraditional hours of work, there are increasing demands for retail and food services to operate on a 24-hour basis in order to support the needs and add to the "quality of life" of these workers (Johnson, et al., 1981).

There is mounting concern over the possible health consequences faced by individuals who work nontraditional hours. Gorden et al., (1986) found that working variable shifts was associated with increased in job stress, use of alcohol and emotional disturbance. Female shift workers also reported higher rates of sleeping pill and tranquilizer use than their day worker counter parts.

The best documented and perhaps most pernicious health problems facing shift workers are disorders of digestion and sleep (Rutenfranz and Knauth, 1976). Disorders of digestion include 
gastritis, gastric and peptic ulcers as well as other intestinal disorders (Shiftwork Committee, 1979). Disturbances of sleep relate both to the duration and quality of sleep (Shiftwork Committee, 1979). Both disorders can, at least in part, be attributed to the necessary alteration of the worker's sleep-wake cycle.

Many physiological functions, such as the fluctuation of body temperature, release of hormones and excretion of metabolites (Minors and Waterhouse, 1981), follow systematic circadian (daily) rhythms that are regulated by a "biological clock" thought to be located in the suprachiasmatic nucleus (SCN) of the hypothalamus (Moore and Klein, 1974; Inouye and Kawamura, 1982). This clock, often referred to as the circadian pacemaker, has an intrinsic period that is greater than 24 hours (Mills, Minors and Waterhouse, 1974; Wever, 1979). Under normal conditions, the pacemaker is entrained to the 24-hour day by social and environmental zeitgebers (i.e,. literally, time-givers), such as meal times (Boulos, Rosenwasser, and Terman, 1980) and the alteration of the light-dark cycle (Czeisler, Richardson, Zimmerman, Moore-Ede and Weitzman, 1981; Rutenfranz and Knauth, 1988).

The circadian system can be thought of as consisting of two components. An endogenous component that consists of one or more oscillators driven by the circadian pacemaker and an exogenous component that consists of environmental and behavioral rhythms. When workers are forced to alter their sleep and activity schedules in order to work at night, there is a conflict between exogenous zeitgebers and the endogenous pacemaker. Consequently, 
physiological rhythms may become desynchronized relative to the usual environmental time cues (Moore-Ede and Richardson, 1985). Moreover, different physiological functions adapt or re-entrain to shifts in the sleep-wake cycle at different rates so there may be desynchronization among various functions as well (Rutenfranz and Knauth, 1988). The result of this desynchronization is that the shift worker may be left in a perpetual state not unlike the temporary state of "jet lag." A similar array of symptoms (e.g., insomnia, malaise, gastrointestinal upset) are experienced by both shift workers and transmeridianal travelers (Moore-Ede and Richardson, 1985).

Because the conventional social rhythm is one of daytime work, evening leisure and nighttime sleep, the individual working night shifts is at odds with most of society. The night worker may be forced to eat, work and sleep at the worst possible times both socially and physiologically (Shift Work Committee, 1979). For example, research indicates that attempting to fall asleep while body temperature is rising or at its maximum (i.e., daytime) is difficult, if not impossible (Czeisler, Weitzman, Moore-Ede, Zimmerman, and Knauer, 1980; Zulley, Wever and Aschoff, 1981). Once the individual is asleep, duration of daytime sleep is significantly shorter than duration of nighttime sleep (Czeisler et al., 1980; Tepas, Walsh and Armstrong, 1981; Zulley et al., 1981). Noise from the environment may also make sleep difficult for the night worker. Traffic noise may wake the day sleeper (Knauth, Rutenfranz, Shultz, Bruder, Romberg, Decoster and Kiesswetter, 1980) and attempting to sleep while others 
in the family are awake may be difficult, especially if small children are present. 


\section{CHAPTER II}

\section{RESEARCH ON THE CIRCADIAN RHYTHMS OF NIGHT WORKERS}

One question that has come out of studies on the circadian rhythms of night workers is whether observed phase shifts in rhythms are maintained when the worker has time off from work. Horne (1985) points out that night workers often will "make up" sleep on days off. This recovery sleep is generally taken in the nighttime hours making sleep longer than usual. Horne contends that this recovery sleep may not be in the best interest of the night worker because it may make subsequent adjustment more difficult. In other words, if the worker reverts to a conventional schedule on days away from work, he or she may find it difficult to resume a nocturnally active schedule when it is time to return to work. Other studies have shown that circadian rhythms rapidly return to diurnality when a diurnal (i.e., daytime) schedule is reinstated (Knauth, Rutenfranz, Herrmann and Poppel,1978; Weitzman and Kripke, 1981).

\section{FIELD STUDIES}

Field studies of shift work have generally not supported long term adjustment of circadian rhythms even in the case of permanent night workers. Van Loon (1963) performed one of the first systematic studies of adjustment to night work and found that 
during 13 weeks of night work the temperature rhythm in workers had merely flattened (i.e., nighttime temperatures showed an increase and daytime temperatures a decrease), and this flattening process reappeared each week. He observed that for one subject this process became more rapid with increased exposure to night work which he inferred as a sign of partial adaptation to night work in this individual. Van Loon suggested that the major factor preventing complete adjustment to the new schedule was the "interruption" in schedule occurring during days off work.

Folkard, Monk and Lobban (1978) studied both full and part time night shift nurses and found that temperature rhythms of the full time staff showed more rapid adjustment over successive night shifts than adjustment seen in temperature rhythms of the part time staff. They attributed this difference to the degree to which full time night nurses scheduled their lives towards night work. However, even though short-term adjustment over successive night shifts was facilitated in the full time staff, no evidence was found for long-term adjustment.

\section{LABORATORY STUDIES}

In the past, as with field studies, many laboratory studies designed to elucidate the effects of shift work on circadian rhythms have focused on the changes in body temperature rhythm following laboratory-induced shifts in the sleep-wake cycle (Folkard, Minors and Waterhouse, 1985). Data from several studies measuring the 
change in temperature rhythm after 8-hour (Colquhoun, Blake and Edwards, 1968a; Knauth et al., 1978) and 12-hour (Colquhoun, Blake and Edwards, 1968b; Weitzman and Kripke, 1981) shifts in the sleepwake cycle have shown that the changed phase position of sleep resulted in a changed circadian phase of body temperature so that nighttime temperatures were significantly increased during the new (night) "work" hours and significantly decreased during (day) sleep hours. Although considerable change in the temperature rhythm had taken place, in none of the studies was the inversion complete by the end of three weeks of a consistent schedule of nighttime wakefulness and daytime sleep.

In general, studies have shown that temperature rhythms do change in response to a shift in sleep time, but that adjustment is slow and incomplete even after several weeks of a strictly enforced nocturnally active schedule with no intervening "nights" off (Colquhoun et al., 1968a, 1968b; Knauth et al., 1978; Weitzman and Kripke, 1981).

\section{MASKING AND THE USE OF CONSTANT ROUTINES}

A main criticism leveled at early studies is that a shift in the temperature rhythm is used to infer a change in the phase of the circadian pacemaker (Minors and Waterhouse, 1988). This inference is problematic because there are two factors that may combine to effect a change in the temperature rhythm. First, part of the change may occur because of an actual shift in the phase of the circadian 
pacemaker. However, at least part of the change may be related to a "masking" effect exerted by environmental or behavioral rhythms (Minors and Waterhouse, 1981; Moore-Ede, Sulzman and Fuller, 1982). For example, it has been well established that body temperature decreases after sleep onset and increases with activity regardless of the timing of these behaviors in relation to the pacemaker (Weitzman, Czeisler and Moore-Ede, 1979). Thus, it can be said that sleep or activity will exert a masking effect on the temperature rhythm. Other rhythms typically used as markers of circadian phase (e.g., cortisol, urinary electrolytes, etc.) are also confounded by sleep and activity. For this reason, results from many of the earlier studies of shift work are difficult to interpret. It cannot be determined how much of the observed change in temperature phase is due to actual re-entrainment of the pacemaker after sleep has been shifted, and how much of the "change" is due to masking effects exerted on the temperature rhythm by sleep or activity.

One way to study the internal clock more accurately is to minimize behavioral or environmental rhythms that lead to masking. Several groups of researchers have attempted this by employing the use of a "constant routine" after a shift in the sleep-wake cycle. Several different protocols have been proposed for the constant routine (Czeisler, Brown, Ronda, Kronauer, Richardson and Freitag, 1985; Mills, Minors and Waterhouse, 1978a; Moog and Hildebrandt, 1986). In all of these constant routine protocols, masking due to thermal changes is avoided by using constant temperature conditions 
and masking due to nutritional variation is controlled by giving subjects small, low protein meals at regular intervals throughout the 24-hour period. The difference in these protocols is that those used by Mills et al. (1978) and Czeisler et al. (1985) did not allow sleep whereas the protocol used by Moog and Hildebrandt (1986) allowed sleep ad libitum. Whether sleep is allowed or completely restricted, it is believed that the use of a constant routine allows a more accurate assessment of the phase of the circadian pacemaker (Minors and Waterhouse, 1988).

In a study performed by Minors and Waterhouse (1988), subjects were studied after their sleep had been delayed by eight hours. Subjects were studied either under a constant routine condition or under a condition in which sleep was allowed. A smaller shift in temperature rhythm was found under constant routine conditions than under conditions in which sleep was allowed. This finding reflects that the circadian rhythm of temperature and other measurements fluctuation are, to some extent, affected or masked by the sleep-wake cycle.

Research using constant routine protocols has added to our understanding of the effects of alterations in the sleep-wake cycle on circadian rhythms, and in general to our understanding of the complexity of the human circadian system. However, it has not been clear how these findings generalize to effects of actual night work. The subjects in these studies are young, fit males who have little experience with night work prior to entering the experiments. The experimental protocols keep the subjects in the lab where they are 
relatively immune to extraneous noise or exposure to outdoor light. Some researchers (Minors and Waterhouse, 1988) question this themselves, commenting "...data from the experimental environment will tend to protect the subjects from some of the consequences of an abnormally timed lifestyle, and it may be this very clash of zeitgebers that contributes to the difficulties experienced by night workers" (pp. 36-37).

Czeisler, Johnson, Duffy, Brown, Ronda and Kronauer (1990) recently reported an attempt to study subjects under more "naturalistic" conditions. In this study, subjects were allowed to travel to and from "work" and sleep at home. The subjects "worked" for four consecutive nights in the laboratory. The "work" nights were bracketed by two 24 -hour constant routines. The baseline phase position of the temperature nadir (trough) was established during the first constant routine and the shift in temperature nadir was determined during the second. The subjects participating in the treatment condition were exposed to high intensity light (similar to the intensity of natural outdoor light just after dawn) between the hours of 2415 and 0745 during "work." The subjects were allowed to return home from the laboratory at 0800 and were confined to total darkness in their home from 0900 to 1700 in bedrooms in which opaque material had been installed over the windows to exclude sunlight. The subjects who participated in the control study "worked" the same nighttime hours in the laboratory but were exposed to ordinary room light rather than high intensity light. Control subjects were not confined to a particular schedule of 
darkness during "nonwork" hours and could sleep at the time of their choosing. The treatment subjects showed an average 9.6 hour phase delay in temperature nadir unlike the control subjects who showed only minimal phase delays or, in several cases, small, maladaptive phase advances in temperature nadir. Although the results of this study seem very promising in the area of rapid adaptation to night work, the design of the study has been criticized (Cambell, Dawson, Eastman and Lewy, 1990; Van Cauter and Turek, 1990). Moreover these results are limited in that they tell us little about what can be expected of long-term night workers.

Clearly then, it seems that there is a compromise between accurately assessing the effects on the circadian pacemaker produced by a shift in the sleep-wake cycle (i.e., with masking effects minimized) and capturing these effects within the complex and often conflicted environment of the night worker. This compromise would not be necessary if a physiological variable could be identified that could be used as a reliable marker for circadian phase position. This marker should have a definite circadian rhythm and ideally, would, be impervious to masking effects.

\section{MELATONIN}

The pineal hormone, melatonin, seems to be just such a marker. The melatonin rhythm is unique in that it is produced at night in all species of animals whether they are diurnal or nocturnal (Aschoff, 1979). Because melatonin has such a stable rhythm, the timing of 
melatonin production is thought to accurately reflect the rhythm of its circadian pacemaker in the SCN (Lewy, 1983). For example when the $\mathrm{SCN}$ is lesioned, the circadian rhythm of $\mathrm{N}$-acetyltransferase, which accurately reflects melatonin synthesis, is obliterated (Moore and Klein, 1974).

Melatonin appears to be unaffected by masking effects of the sleep-wake cycle (Hoban et al., in press), sleep deprivation (Jimmerson, Lynch, Post, Wurtman and Bunney, 1977; Akerstedt, Froberg, Friberg and Wetterberg, 1979), diet (Arendt, 1979; Herbert and Reiter, 1981) or stress (Illnerova, 1976; Klein and Parfitt, 1976; Lynch, Eng and Wurtman, 1973; Vaughan et al., 1978; Vaughan, Bell and De La Pena, 1979). Other than specific drugs that affect the amplitude of melatonin secretion, it is masked or blocked only by bright light (Lewy, Wehr, Goodwin, Newsome and Markey, 1980). Therefore, if bright light is avoided, the melatonin rhythm provides an accurate reflection of the rhythm of its circadian pacemaker. In subjects with normally entrained circadian rhythms, the onset of melatonin production occurs between 1900 and 2300 and the duration of production is approximately 12 hours. The timing of melatonin production can be determined with a high degree of resolution ( $\pm 15 \mathrm{~min}$.), especially the daily onset (Lewy, Sack and Singer, 1984).

Waldhauser, Vierhapper and Pirich, (1986) studied melatonin rhythms in two night workers and found that the workers' melatonin production occurred during daytime hours. However, the night shift 
was unconventional (i.e., 1900 to 0400) and no sleep data were collected.

\section{PERSONALITY}

It has been suggested that certain personality types may adapt more readily to night work. For example, Horne and Ostberg (1976) have devised an English version of their Morningness-Eveningness questionnaire which is used to evaluate the degree to which an individual can be thought of as a "lark" (morning type) or an "owl" (evening type). Using a Swedish version of this instrument adapted for shift work, Ostberg (1973) found that morning types did not adapt their habits to the needs of shift work as readily as evening types, and that morning types have more autonomous (i.e., less flexible) circadian rhythms.

Other studies have shown that introverted subjects show superior performance at visual inspection tasks in the early morning, whereas extraverted subjects perform better later in the day (Colquhoun, 1960; Colquhoun and Cocoran, 1964) and that these performance differences are, to some extent, associated with differences in the circadian phase of body temperature [i.e., individuals scoring toward introversion have relatively advanced temperature rhythms and individuals scoring toward extraversion have relatively delayed temperature rhythms (Blake, 1967).]

There is some evidence (Lund, 1974) that individuals with high neuroticism scores show a greater tendency for "internal 
desynchronization" of rest-activity and body temperature rhythms under conditions free from zeitgebers (i.e., temporal isolation). Although night workers do not live or work in an environment free from external time cues, they are often exposed to inconsistent time cues and night work inherently results in conflicts among zeitgebers. 
CHAPTER III

\section{RATIONALE AND OUTLINE OF THE PRESENT STUDY}

The present study attempted to discover the degree of circadian adjustment that night workers make following an extended period of time on the night shift. No attempt was made to alter or control the subjects' sleep-wake or light-dark schedules prior to the measurement of circadian phase position. Rather, it was believed that by evaluating experienced permanent night workers, under their normal circumstances, with all of the physical, social and environmental conflicts they may be expected to encounter, an accurate and ecologically valid picture of physiological adaptation, or lack thereof, could be described.

\section{PHYSIOLOGICAL ADAPTATION}

The first question the present study attempted to answer was whether long-term, permanent night shift workers when compared to day workers would show a difference in circadian phase of melatonin production which would be indicative of adjustment to a nocturnally active schedule. If physiological adjustment does occur with long-term night work, it was expected that this adjustment would be reflected in the timing of the onset of melatonin production. Specifically, it was expected that the melatonin onsets of 
the night workers would occur in the morning hours prior to the initiation of daytime sleep, whereas the melatonin onsets of the day workers would occur in the evening hours prior to the initiation of nighttime sleep. In addition to assessing the interval between the onset of melatonin production and the onset of sleep, physiological adjustment to a night work schedule could also be assessed as the degree to which work hours occurred during "biological daytime" (i.e., the time free from melatonin production) and the degree to which sleep occurred during "biological nighttime" (i.e., in conjunction with melatonin production).

If night shift workers were successful in achieving physiological adaptation to their nighttime schedules, it was predicted that there would be little ill effect on their state of health or physical wellbeing. Conversely, if physiological adjustment to a nocturnally active schedule was not achieved, it was predicted that night workers would report problems with sleep. Sleep would be expected to be inferior both in quality and duration when compared to sleep reported by day workers. If sleep was found to be inferior, it was also expected that night workers would attempt to "make up" lost sleep on their days away from work and that a considerable portion of this "make up" sleep would occur during nighttime hours when off duty. 


\section{PSYCHOLOGICAL ADAPTATION}

Because so many factors other than hours of work can enter into psychological adjustment and satisfaction with one's job and lifestyle, it could not be said for certain that night workers would show increases in emotional disturbance or lack of satisfaction due only to their schedule. However, because lack of physiological adjustment to night work is defined by complete or partial desynchronization and internal desynchronization has been associated with neurosis (Lund, 1984), it was expected that subjects who showed a lack of physiological adaptation to night work would score high on neurosis. Likewise, it was hypothesized that a lack of physiological adaptation would be observed in night workers who scored toward the "morningness" end of the continuum.

For the purposes of the present study it was expected that lack of physiological adaptation to night work would also be exhibited in greater emotional disturbance and decreased satisfaction with work and/or life-style. 
CHAPTER IV

\section{METHODOLOGY AND PROCEDURES}

\section{SUBJECTS}

\section{Night Workers}

Ten subjects were recruited from employees working the night shift at Oregon Health Sciences University (OHSU) and other hospitals and industries in the area via informational posters and advertisements in employee newsletters. One subject was unable to complete the protocol, leaving a total of nine subjects in the data analysis. Subjects had been working a regular night shift for at least six months. The length of employment on the night shift ranged from 7 to 60 months; the median length of employment was 15 months. The seven women and two men ranged from 22 to 47 years of age, with an average age of $35(S D=8.5)$ years.

Upon giving informed consent to participate in the study, subjects were interviewed to ensure that they were in good physical health and were not currently taking any medications that are known to affect melatonin production (e.g., clonidine, propranolol). Subjects were paid a $\$ 25$ honorarium for their participation in the study. 


\section{Day Workers}

Control data were collected from nine individuals who had been recruited through both local newspaper advertisements and the OHSU campus newsletter to participate as normal control subjects in a winter depression study taking place concurrently with the study of circadian rhythms in night workers. In order to be accepted as a normal control subject in the winter depression protocol, subjects needed to be in good physical and mental health and could not be taking any medications known to affect melatonin production. These subjects had not participated in work that included a night shift duty for at least three months prior to the time of data collection. The seven women and two men ranged from 29 to 68 years of age, with an average age of $44(S D=12.7)$ years.

\section{PROCEDURES}

\section{Night Workers}

The nine night shift workers were admitted to the Clinical Research Center (CRC) at OHSU for a 24-hour period following their last shift of the week in order to collect melatonin onset data. Data were collected during the winter months between January 6 and February 25. Four of the night workers had worked five nights prior to their admission, three had worked four nights and one worked only three nights prior to admission. This reflected these subjects' regular work schedules. One of the subjects was ill three days prior to admission and had been back to work only two nights prior to 
admission. Subjects were not confined to any particular sleep-wake schedule or light-dark schedule prior to their admission for determination of melatonin onset. While in the CRC, subjects were confined to a dimly lit room so that melatonin production would not be suppressed by ambient light. Subjects were allowed to eat and sleep ad libitum. An intravenous catheter was inserted into a forearm vein and a $5 \mathrm{ml}$ blood sample was obtained once every hour for 24 hours.

\section{Day Workers}

As in the study of night workers, the winter depression protocol involved subjects coming to the Clinical Research Center at OHSU for blood sampling. Samples from this group were collected during the first week of January. Because melatonin production in normal individuals occurs exclusively at night, blood samples were taken every half hour between 1800 and 2300 in order to determine the timing of the onset of melatonin secretion. In addition to compensation for their participation in the winter depression protocol, these subjects were paid an honorarium of $\$ 5$ for completing questionnaires that were not part of the winter depression protocol. 


\section{Procedures Common To Both Groups}

Sleep times and quality as well as energy levels, sleepiness, mood state, anxiety level and light exposure were assessed for the twoweek period prior to the blood sampling procedures (data from two day workers included only one week) by way of daily diaries kept by subjects and by items pertaining to sleep pattern taken from the General Health and Adjustment Questionnaire (Tasto, Colligan, Skejei and Polly, 1978). Measures of satisfaction with sleep from the dayto-day subjective sleep quality ratings in which subjects rated each sleep period on a five point scale (i.e., 1 = very good, $2=$ good, 3 = fair, $4=$ poor, $5=\mathrm{bad}$ ) focused on how sound and refreshing sleep was. The one-time rating in which subjects rated their sleep on a four point scale (i.e., $1=$ excellent, $2=$ good, $3=$ fair, $4=$ poor) was more general in that it inquired about the general pattern of the subjects sleep. A sample of the daily diary and instructions to subjects are shown in Appendix A. Average sleep onsets/offsets, quality and total sleep times (with and without naps) were calculated separately for days at work and days away from work for the two weeks prior to the night workers' admission for melatonin determination. Naps, when they occurred were defined as the shorter of two sleep episodes. Sleep measures for day workers were collected in much the same manner except that there was no differentiation made between days at work and days away from work (i.e., the averages included weekends). Although napping was not assessed on a day-to-day basis for day workers they were 
questioned about their usual sleep pattern including whether or not they took naps.

While subjects were confined to the CRC, they completed several paper and pencil measures. The General Health and Adjustment Questionnaire (Tasto et al., 1978), was also completed by all research participants. This questionnaire is extensive and includes items inquiring about satisfaction with work, social life and leisure time activities as well as items pertaining to general health, mood, sleep and demographic data. In order to determine if night workers differed from day workers in their usual day/night orientation for "best" functioning, subjects completed the Morningness-Eveningness Questionnaire (Horne and Ostberg, 1976).

Subjects completed two psychological measures: The Profile of Mood States (POMS) (McNair, Lorr and Droppleman, 1971) and the Eysenck Personality Inventory (EPI) (Eysenck and Eysenck, 1968). The 65 POMS adjectives purport to measure six identifiable mood or affective states: Tension-Anxiety, Depression-Dejection, AngerHostility, Vigor-Activity, Fatigue-Inertia and ConfusionBewilderment. A total mood disturbance (TMD) score is obtained by summing the scores across all six factors (weighting Vigor negatively). The POMS was developed through six factor-analytic studies and normed on a psychiatric outpatient population as well as a population of undergraduates at a large eastern university. The POMS appears to be internally consistent with inter-item reliabilities ranging from .87 on the confusion factor to .95 on the depression factor. Test-retest reliabilities of the POMS were measured from 
intake to pretherapy in the outpatient population and range from .65 for the vigor factor to .74 on the depression factor. While these coefficients are rather low, the authors argue that the stability of a fluctuating state such as mood should not be expected to reach levels achieved by measures of personality, and in fact, high coefficients of stability could indicate a lack of construct validity. The POMS appears to possess moderately good predictive and construct validity (McNair, Lorr and Droppleman, 1971). All of the independent POMS factors show significant correlations with both a modified version of the Hopkins Symptom Distress Scales and the Taylor Manifest Anxiety Scale. Because all POMS factors, with the exception of anger, correlate significantly with gender, separate norms are given for males and females.

The Eysenck Personality Inventory (EPI) measures personality in terms of two pervasive, independent dimensions. These dimensions are identified as extraversion-introversion (E-I) and neuroticismstability (N-S). Each of these traits is measured by means of 24 questions, selected on the basis of item and factor analyses, to which the examinee answers "Yes" or "No." A response distortion (Lie) scale is also included to detect attempts to falsify responses. The EPI is internally consistent with split half reliabilities of the parallel forms reported as .75 for the E-I dimension and .89 for the N-S dimension. Test-retest reliabilities were measured in two normal populations, one at nine months; the other at one year. For E-I reliabilities are .85 after nine months and .80 following a year. For N-S reliabilities are .91 after nine months and .81 following a year. The dimensions of 
the EPI are independent $(r=-.09)$ and although the EPI does not correlate with gender, it is significantly related to age. There is a significant trend for both Extraversion and Neurosis to decline with age. The scales of the EPI show high correlations $(.79-.92)$ with other measures purporting to measure the same dimensions.

\section{Analysis of Physiological Data}

Blood samples were drawn by nursing personnel skilled in phlebotomy. Blood samples were immediately refrigerated and within 24 hours, samples were centrifuged, plasma was separated from the cells and frozen for later analysis. Serial plasma samples were assayed in order to determine melatonin concentration. The dim light melatonin onset (DLMO) was defined as the point at which plasma melatonin concentration first reaches $10 \mathrm{pg} / \mathrm{ml}$ and continues to rise. One subject (N6) appeared to have a relatively high baseline of melatonin production (i.e., levels did not drop below $10 \mathrm{pg} / \mathrm{ml}$ for more than a few hours). Therefore, the timing of this subject's DLMO was determined using the criterion of $20 \mathrm{pg} / \mathrm{ml}$. Plasma samples were analyzed for melatonin content by gas chromatographynegative chemical ionization mass spectrometry (Lewy and Markey, 1978) a method which is recognized as the most sensitive and accurate of the melatonin assays (Arendt, 1981; Lynch, 1983; Rollag, 1981). 


\section{CHAPTER V}

\section{RESULTS OF THE STUDY}

\section{PHYSIOLOGICAL AND BEHAVIORAL MEASURES}

\section{Dim Light Melatonin Onsets}

It was hypothesized that physiological adaptation to night work would be reflected in the circadian phase position (i.e., time of day) of the onset of melatonin production. Specifically, it was hypothesized that the onset of melatonin production in night workers would show a diurnal (i.e., daytime) orientation, occurring in conjunction with daytime sleep, whereas the onset of melatonin production in day workers would show a nocturnal (i.e., nighttime) orientation occurring in conjunction with nighttime sleep.

With the exception of one subject (N7), whose melatonin onset occurred at 24.50, (all times are presented in "military" time with minutes expressed in hundredths of an hour) the first hypothesis that night workers' DLMOs would occur during daytime hours, was supported to a great extent. The night workers' DLMOs ranged from 10.47 hours to 24.50 hours. The day worker DLMOs ranged from 18.80 hours to 22.80 hours. The medians of the two groups were significantly different, with the median DLMO for night workers occurring at 14.47 hours and the median DLMO for day workers occurring at 21.30 hours $(p \leq .001$, Mann-Whitney $U$ test). Moreover, 
the onset of melatonin production in night workers was not only statistically different from day workers, night workers as a group showed a clear diurnal orientation of melatonin production as opposed to the nocturnal orientation normally observed. However, there was greater variability in the timing of melatonin production among night workers when compared to the group of day workers and the typical temporal relationship of the onset of melatonin production occurring prior to the onset of sleep was not preserved. Figure 1 shows the relationship between the onset of melatonin production and average sleep periods for individuals in both groups.

Due to the variability in the timing of both the onset of and duration of melatonin production among night workers, separate case summaries for the night workers are presented in Appendix E. The case summaries are numbered consecutively from N1 to N9. Subject N1 showing the "best synchronization" among the timing of melatonin production, sleep and work, and subject N9 showing the "least synchronization." Optimal synchronization among these variables would be defined by sleep periods occurring during the time of melatonin production as well as by a lack of melatonin production during work times. Although unlikely, it is possible that the timing of light exposure on the day prior to measurement of melatonin production may have had impact on the observed phase position. Therefore in Figure 2, graphic representations of night workers' DLMOs are shown with sleep periods proximal to the day of measurement. 


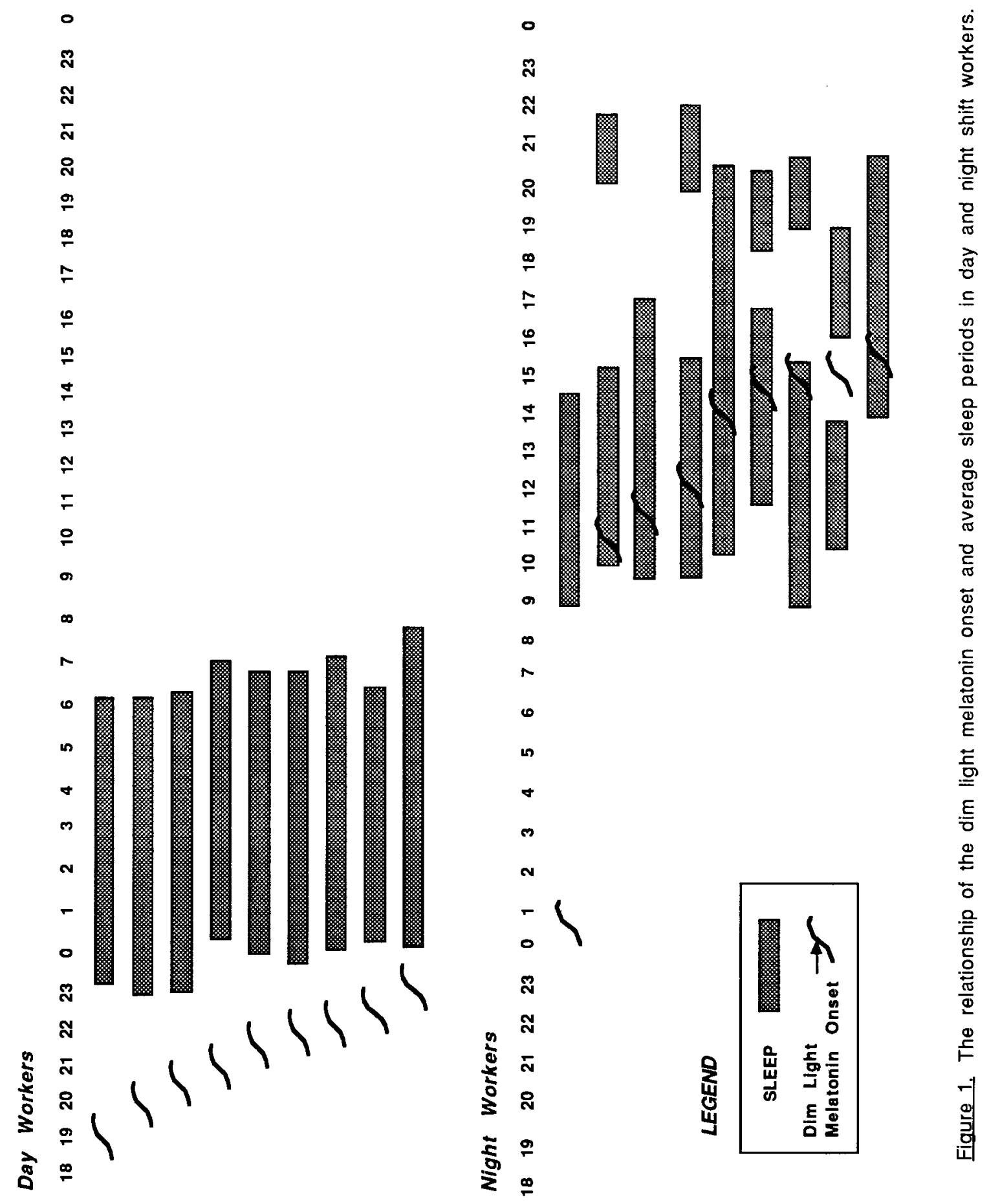




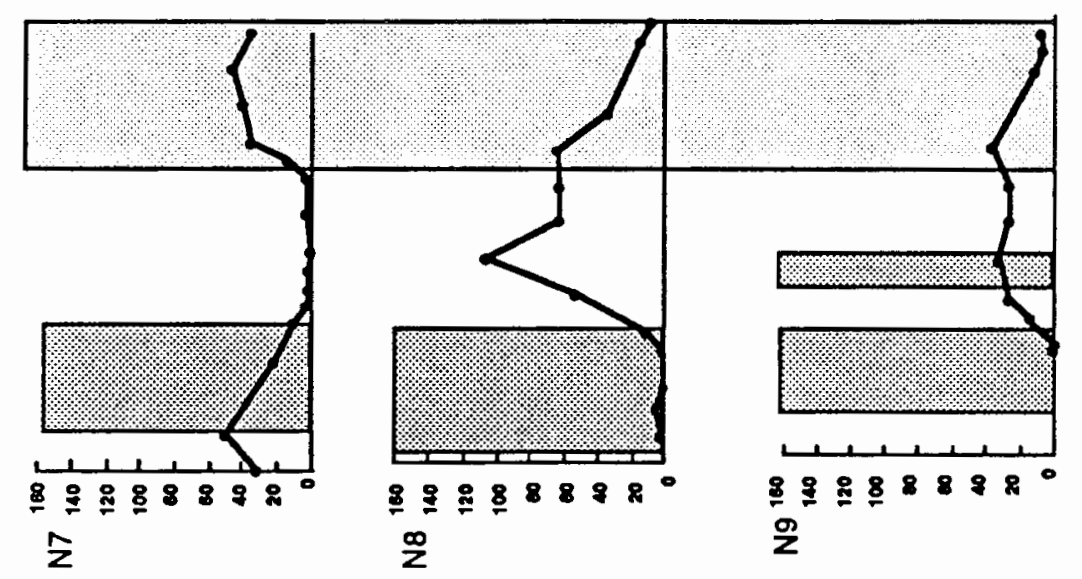

$\mathbf{8}$

$\pm$

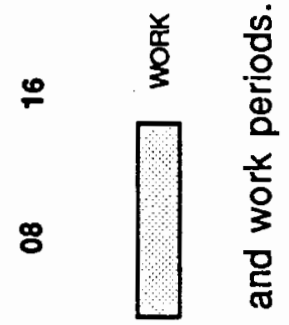

乏

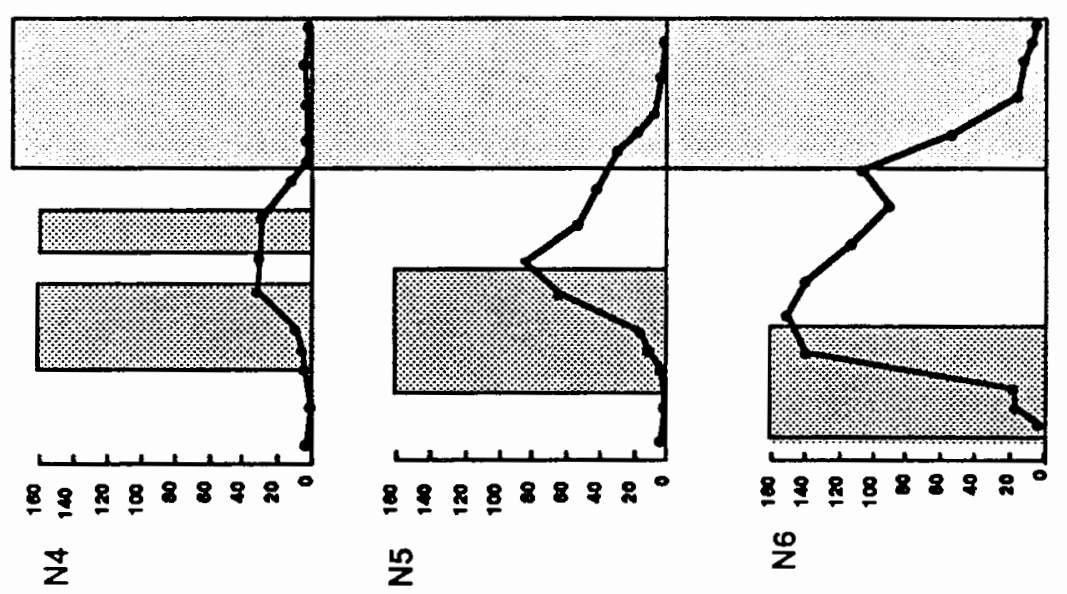

\&

$\frac{\text { 응 }}{\mathbb{\Phi}}$

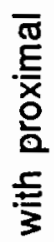

ส

.

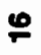

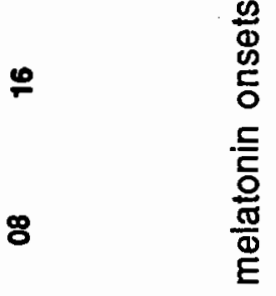

$8 \quad \frac{. \frac{.}{\delta}}{\frac{\sigma}{\sigma}}$

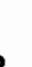

*

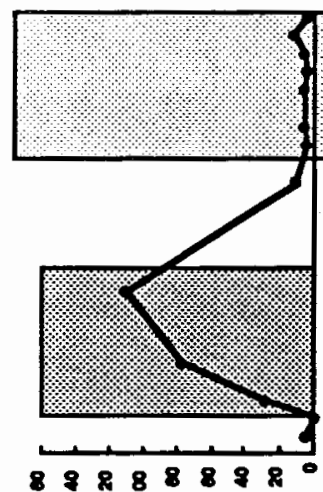

$89988898^{\circ}$

$\bar{z}$

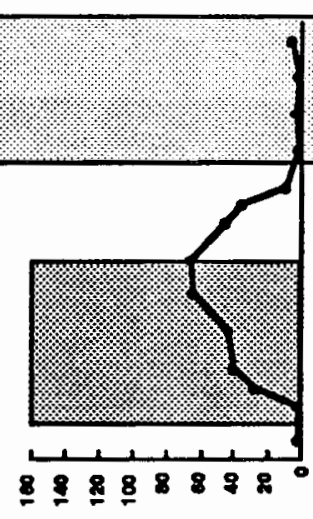

₹

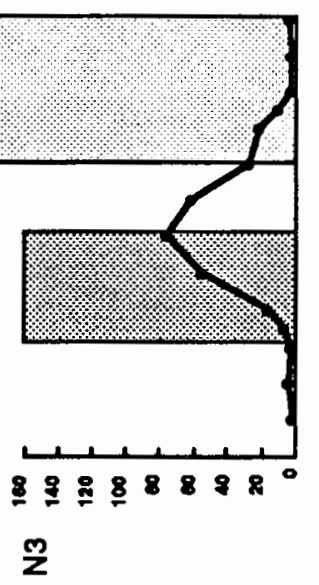

₹

(|u/bd) u!uołejəw 
Sleep

Results of the self-report sleep diary data revealed that day workers obtained more average total sleep (median $=7.7$ hours of sleep per night, range $=1.8$ hours) compared to the night workers' average main sleep periods (median $=6.0$ hours, range $=4.9$ hours) ( $p \leq .05$, Mann-Whitney $\mathrm{U}$ test). However, if night workers' naps were included in their average totals, (median $=7.0$ hours, range $=4.4$ hours) there was no significant difference between group medians of average total sleep obtained.

The sleep patterns of day workers were very consistent as a group and within subjects over time, with the average time of sleep onset at 23.42 ( $S D=0.58$ hours) and average sleep offset at 06.92 ( $S D=0.71$ hours). The night workers in the present study utilized a variety of sleep strategies, with some individuals sleeping soon after work and others remaining awake for several hours following work. Some night workers routinely took naps in addition to longer main sleep periods (although the naps and main sleep periods did not always occur in the same temporal order); other night workers did not nap at all. Sleep onset and offset times among and to some extent within individual night workers varied so greatly that average sleep onset and offset times for this group are fairly meaningless. Therefore, graphic representations of sleep patterns for individual night workers prior to the determination of melatonin onset are presented in Appendix B.

The group of night workers showed a difference in the total amount of sleep obtained on work days (median $=7.0$ hours, 
range $=4.4)$, compared with the total length of sleep on days away from work (median $=9.0$ hours, range $=8.1$ hours $)(p \leq .005$, Wilcoxon signed rank test). Thus, night workers increased total sleep time during days away from work. Figure 3 shows the percentage of day and night workers asleep at any time during an average 24-hour period. The percentages of night workers asleep at any given time are presented both for days at work and days away from work. None of the night workers maintained an orientation of daytime sleep and nighttime wakefulness on their days off, tending to make up sleep either by napping in the afternoon or sleeping extra hours in the evening.

When subjects were asked the difference between how many hours of sleep they would like to have and the amount they actually slept, both groups reported obtaining less sleep than desired. However, night workers reported larger subjective sleep deficits ranging from no deficit to two hours than day workers who reported sleep deficits ranging from no deficit to one hour.

There were no differences between day and night workers in subjective ratings of daily sleep quality nor were there differences between sleep quality ratings made by night workers for sleep 


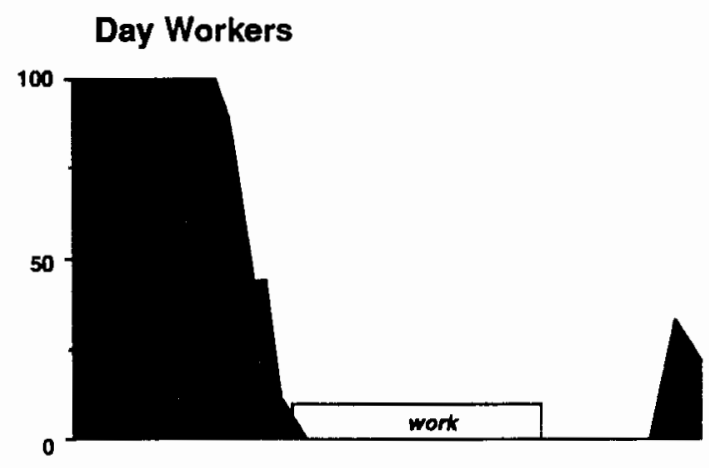

Night Workers

(days away from work)

$\frac{0}{0}$
$\frac{0}{0}$
$\frac{0}{2}$
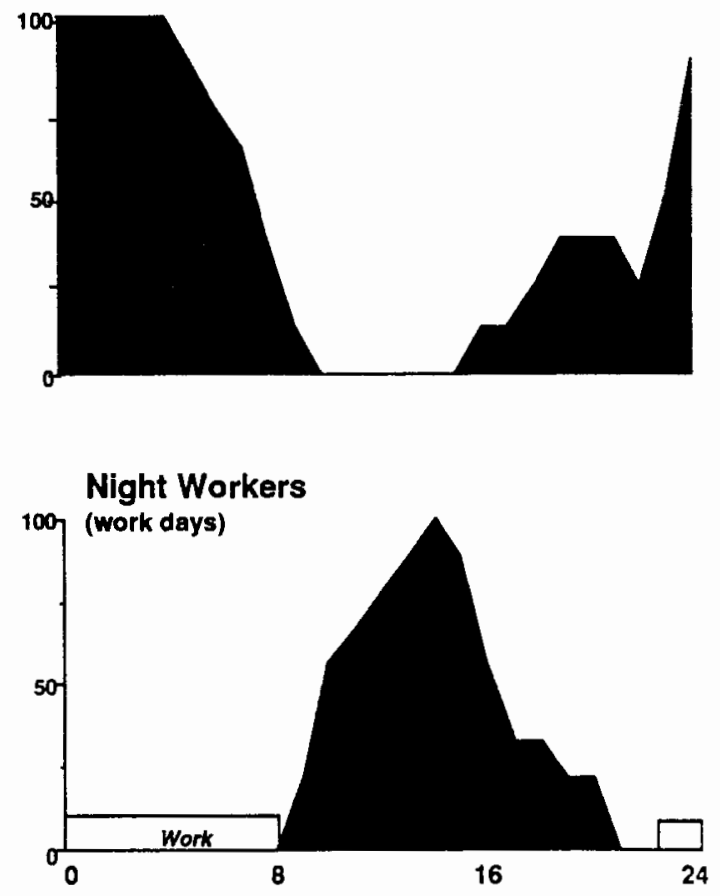

Time of day

Figure 3. Percentage of day and night shift workers asleep during an average 24-hour period. 
during days away from work compared to those made for sleep during days of work. Night workers rated their average daily sleep quality as "good" and day workers rated their average daily sleep quality between "good" and "excellent" on a five point scale from "excellent" to "bad." However, when subjects were asked to give a rating of their sleep pattern in general, one night worker (N8) rated her sleep pattern as "poor," with the average rating for the night workers falling between "fair" and "good." Because these ratings were made on different scales, little can be said about how they might be related. In addition a rating of satisfaction with general sleep pattern may not be the same as a day-to-day sleep quality rating. It is likely that sleep quality is inherent in a rating of satisfaction with general sleep pattern, but that other issues may enter into satisfaction with sleep pattern as well, such as how the sleep pattern allows for or disrupts social interaction.

It was hypothesized that failure to physiologically adapt to a night work schedule would be apparent in inferior quality and quantity of day sleep episodes. Although some of the night workers in this study clearly slept better than others, as a group night workers obtained as much total sleep as the day workers did and rated their quality of sleep comparably to the day workers.

It was also hypothesized that lack of physiological adaptation would be exhibited in attempts by night workers to make up sleep on their days off. Apparently many of the night workers in the present study felt the need to make up sleep on days away from work, sometimes sleeping in excess of 12 hours in a single episode. 
For some individuals, (N1, N3, N6) sleep duration on days off increased by as much as an average of four to six hours.

\section{PSYCHOLOGICAL MEASURES}

\section{Profile of Mood States}

Night workers tended to score higher than day workers on the total POMS with median total scores of 19 and 8, respectively $(p \leq .01$, Mann-Whitney $\mathrm{U})$. All of the day workers and the majority of night workers scored within the normal range on the individual POMS factors. However, due to the high degree of variability among night workers, individual POMS scores are given in the night workers' case summaries (Appendix E).

\section{Eysenck Personality Inventory}

Night and day workers did not differ on tendency toward introversion-extraversion. However several night workers (N4, N6, N8 and N9) scored above the normal range (between the 31st and 70th percentiles) on the neuroticism dimension. Scores on the lie scale were within normal limits for all night workers. All of the day workers scored within or below the normal range on the neurosis scale of the EPI. However, two of the day workers who scored very low on this dimension also had elevated scores on the lie scale, suggesting that scores for these two subjects may be unreliable. 


\section{Morningness-Eveningness Questionnaire}

None of the nine day workers were classified as evening type.

Four of the day workers were classified as morning type, with two scoring as "definite" type and two scoring as "moderate" type. The remaining five day workers scored in the median range of the scale and were classified as "intermediate" type.

Of the nine night workers, two were classified as "moderate" evening type, two were classified as "moderate" morning type, with the remaining five scoring in the median range of the scale classifying them as "intermediate" type. Although it cannot be said what, if any, clinical importance the differences between the two groups on this scale may hold, if night workers are considered on a case-by-case basis, some interesting "patterns" begin to emerge, especially with respect to differences in sleep strategies.

The two night worker subjects who scored as moderately morning types (subject N3 and subject N8) had different sleep strategies with markedly different results. These data are presented graphically in Figure 4. Subject N3 routinely retired late in the day $(M=13.75)$, slept an average of 6.13 hours and did not take naps. He rated his overall sleep pattern as "excellent" and other than complaining that he was not able to sleep with his wife often enough, he had no other complaints with regard to sleep.

In contrast, $\mathrm{N} 8$ (the other moderately morning type), generally attempted to initiate sleep soon after the end of the night shift. This subject exhibited a fairly "chaotic" sleep schedule. Not surprisingly, 
Subject N3

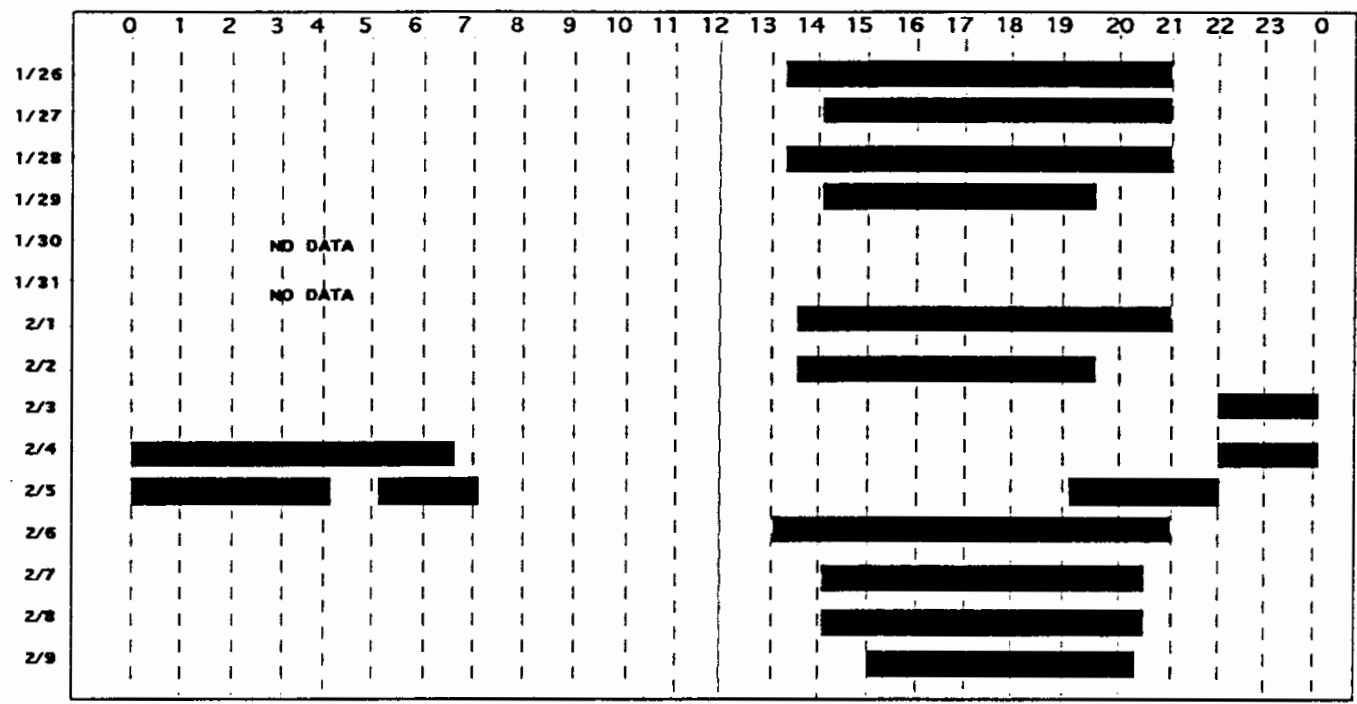

Subject N8

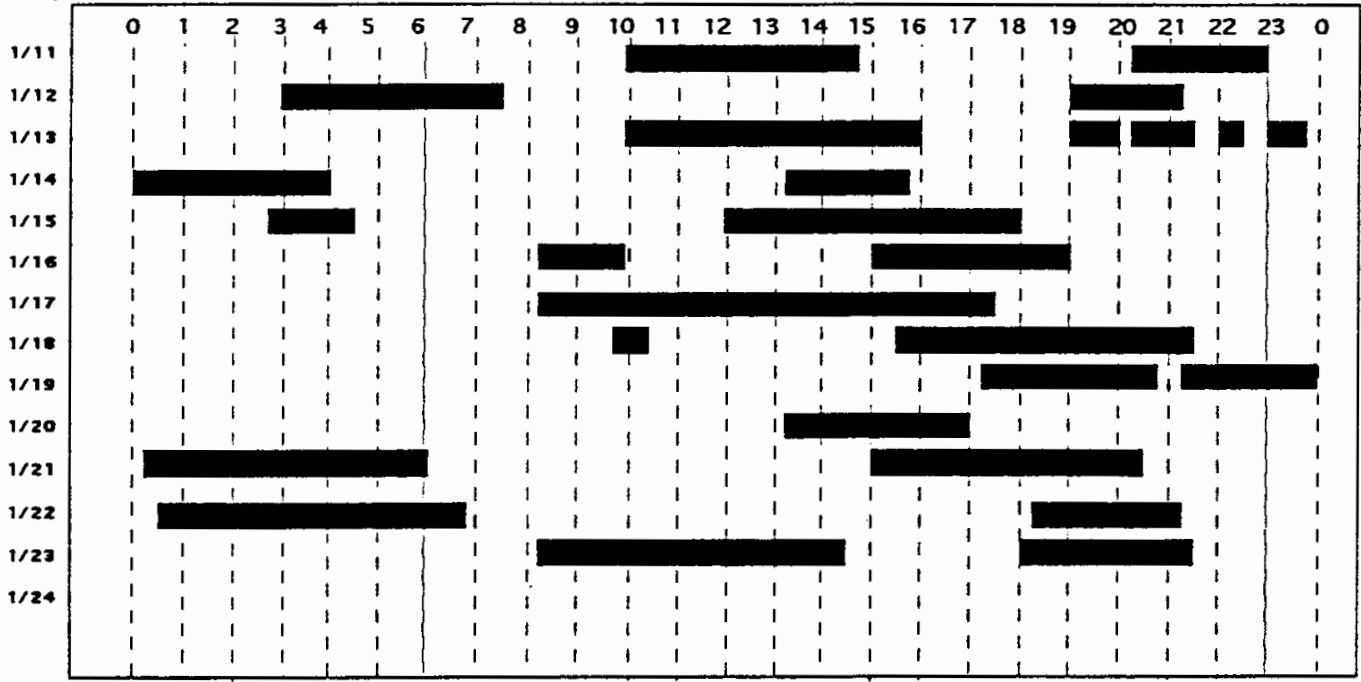

Sleep

Figure 4. Sleep strategies in two "moderately morning" type night workers. 
subject N8 rated her overall sleep pattern as "poor," and complained of frequent insomnia and feeling sleepy upon awakening, during work and after work. She did not report using sleep medications per se, but did report the occasional use of alcohol as a soporific. Although subject N8 scored in the average range on the POMS factors, she scored second to the highest on the neurosis score on the EPI (89th percentile).

Only two night workers (N5 and N7) were classified as moderately evening types. Both of these individuals initiated sleep prior to 10.00 and did not nap, however, these were the only similarities in these two subjects' sleep. N5 rated her overall sleep pattern as "good" and slept the longest of any of the night workers, sleeping an average of 10 hours on work days and 11 hours on her days off. Subject N7 slept the shortest of any of the night workers, obtaining an average of 5.5 hours on work days and 5.25 hours on days off and complained of frequent insomnia and the frequent use of sleep medications. In some respects N7 showed the least physiological adaptation of any of the night workers. Her melatonin onset, although somewhat delayed, was in a fairly normal phase position (24.50), suggesting that she had not been successful in adapting to a nocturnally active schedule. However, this subject's melatonin production was abnormally long in its duration (15.5 hours). This apparent lengthening of physiological nighttime allowed her to sleep during the time of melatonin production, yet it also required that she work during this time. 
There are several possible suggestions for why N7 did not show much, if any physiological adaptation. One possibility is that she was the least experienced night worker in the group having worked only 7 months on the night shift prior to her inclusion in the study. It is also possible that her fairly regular employment at a different job in the afternoon had an effect on her ability to adapt. It was surprising that this subject appeared to be one of the better adapted subjects with regard to psychological well-being.

\section{General Health and Adjustment Questionnaire}

There were no major differences in subjects' health histories, with both groups being relatively free of chronic disease. One night worker reported problems with a hernia, one complained of asthma, one of gall bladder trouble and thyroid abnormalities and two reported problems with insomnia. Of the day worker group, one individual reported being troubled by asthma, three complained of being bothered occasionally by arthritis and one had thyroid abnormalities. Workers ranked the frequency of 43 physical symptoms (see Appendix C) on a 4-point ( 1 = never, 2 = occasionally, 3 = frequently, $4=$ constantly) scale and a total score was calculated for each subject. The medians of the total scores were different for the two groups ( $p \leq .05$, Mann-Whitney $U$ test); the median for night workers was 60 (range $=21$ ) whereas the median total score for day workers was 52 (range $=16$ ). For the most part, chronic physical symptoms were not frequent in either group, 
however symptoms reported by night workers are included in the individual case reports in Appendix E.

\section{Life Satisfaction}

The 29 questions related to lifestyle satisfaction are shown in Appendix D. A total score was calculated for each subject and the medians of the two groups were compared. The night workers had a median total score of 57 (range $=29$ ) whereas the day workers had a median total score of 41 (range $=44$ ). Although the medians are significantly different ( $p \leq .05$, Mann-Whitney $U$ test), the ranges of total scores were rather high and the medians for both groups were well below the highest possible total score of 124 , suggesting that overall, both groups were moderately satisfied with lifestyle.

\section{Self-Reported Daily Ratings}

Night workers daily ratings of energy, sleepiness, anxiety level and mood state were very similar to ratings made by day workers. All workers rated these variables as consistently average or better than average. The late timing of dawn during the winter months when this investigation took place, coupled with relatively short work-to-home travel times, resulted in the majority of subjects receiving relatively little light exposure on their way home. Three subjects (N1, N7 and N9) returned home prior to dawn. Of the remaining six subjects, five (N2, N4, N5, N6 and N8) were exposed to less than 30 minutes of light exposure on their way home and one (N3) was exposed to 45 minutes of light. Only subject N3 reported 
outdoor light exposure after reaching home, all others reported exposure to only dim or average room light prior to sleep. 


\section{CHAPTER VI}

\section{CONCLUSIONS AND RECOMMENDATIONS}

To date, much of the research examining the circadian rhythms of night workers has suggested that little if any long-term, physiological adaptation to a schedule of nighttime activity and daytime sleep is possible. The exposure to bright outdoor light on the night worker's return home from work and the tendency for night workers to revert to a day active schedule on days away from work have been suggested as explanations for the lack of complete adaptation. Despite this, nearly all of the subjects investigated in the present study had melatonin rhythms that were, to varying degrees, synchronized to a nocturnally active schedule.

Although some individual night workers had complaints with regard to sleep, as a group the night workers in the present study did not differ from the day workers in self-reported quality or quantity of sleep obtained on work days. Taken together with the finding that night workers did not rate the quality of sleep during days off differently from sleep during work days, adds further to the contention that the group of night workers evaluated in the present study show a greater degree of physiological adaptation to their schedules than has been previously found. 
It is possible that self-reported sleep times and sleep quality were inaccurate. Although great care was taken to ensure that subjects understood the procedures, it may be that individuals are not very good at estimating these variables. An objective measure of sleep time and quality such as electronic activity monitoring or polysomnography would have added to the reliability and validity of these data. Another possible limitation of this study is that the day workers in this study were an average of 10 years older than the night workers and so may have been experiencing some decline in sleep quality and quantity related to the natural aging process. It is possible that significant differences would have been found both in quality and duration of sleep had the night workers in this study been compared to a group of day workers who were matched more closely on age. However it is important to note that both day and night workers in this study obtained fairly normal amounts of sleep per 24-hour period (7.5 hours and 7.0 hours respectively) and generally rated their sleep quality as good to excellent.

While the results of the present study show a considerable degree of synchronization among sleep, work and the circadian phase of melatonin production, suggesting that at least some individuals are able to adjust physiologically to a schedule of nighttime work and daytime sleep, the present study did not address how this adjustment occurs or if the adjustment is stable over time. Future investigations in this area need to address this issue by measuring melatonin production in night workers longitudinally. Because most night workers revert to a fairly normal schedule of nighttime sleep 
and daytime wakefulness on their days away from work, measurements should be made both after the workweek, as was done in the present study as well as following the workers' days days away from work. This would help to establish whether and to what extent the circadian pacemaker has to readjust when the night worker returns to work.

Although the night workers as a group reported no more problems with sleep than the control group of day workers, several individuals reported unsatisfactory sleep and incidences of insomnia. This may be due in part to the unexpected finding that there was a tendency for the night workers' melatonin production to begin after the initiation of sleep so that sleep occurred at an "early" circadian phase suggesting that physiological adaptation for some night workers remains less than perfect. Why is the melatonin rhythm (and by inference the circadian pacemaker) delayed with respect to sleep? The phase response curve (PRC) for the effects of light exposure in humans, (Czeisler et al., 1989; Wever, 1989) predicts that bright light exposure in the first part of the subjective night will delay the circadian pacemaker whereas bright light in the last half of the subjective night will advance the circadian pacemaker. Because this investigation took place during the winter months, some of the subjects returned home from work prior to dawn and so received no bright light. Indeed, only one subject received more than 45 minutes of bright light in the morning after work. The remaining night workers were exposed to only ordinary or dim indoor light from the time they returned home until the time they went to sleep. Once 
asleep, they slept through the remaining daylight hours and therefore received little, if any, bright light exposure during the afternoon hours prior to dusk. Therefore, it seems likely that subjects in the current study received insufficient light on the phase advance portion of their PRCs and whatever bright light they did receive, fell on the phase delay portion of the PRC. The end result of this set of circumstances would be a tendency for the circadian phase of melatonin to become "overdelayed" with respect to sleep.

There are several suggestions that could be made which might help these individuals achieve even better adaptation than that which has already occurred. First, since in most cases the melatonin onset is abnormally delayed with respect to sleep, a simple solution would be to prescribe a later bedtime so that the night worker is sleeping more in phase with melatonin production. Incidentally, this is a schedule that more closely follows the normal temporal order of day workers (i.e., awakening, working, recreation and then sleep). However, many night workers would resist this schedule because it would interfere with family and other activities that normally take place in late afternoon or early evening thus leading to additional social isolation. In addition, work schedules are generally inflexible, this approach would not correct the extension of "biological nighttime" into the work hours.

Another, more complicated, approach would be attempting to align the circadian pacemaker so that "biological nighttime" coincided more closely with the preferred sleep time and "biological daytime" occurred during work hours. The data from the present study 
suggest, that at least during the short winter months, night workers have melatonin rhythms that are somewhat over delayed. It follows then, that a corrective phase advance of the circadian pacemaker in the range of one to four hours would be beneficial.

One way to achieve an advance in the pacemaker is by the application of appropriately timed exposure to bright light. The recent findings of Czeisler et al. (1990) have shown that the circadian pacemaker may be quickly delayed by the application of appropriately timed exposure to bright light (and appropriately timed avoidance of light) in such a way as to facilitate rapid adaptation to a nocturnally active schedule. However, their findings from the no-treatment condition also imply that without such a regimen night workers left on their own would not adjust to a schedule of nighttime work and daytime sleep. This implication is problematic on several counts. On a practical level, it is unlikely that individuals who work at night on a regular basis, even with great motivation could or would follow such a regimen on a long-term basis. On a physical level, the results of the present investigation suggest that some adjustment does occur in long-term night workers and that during the short daylight hours of winter night workers may actually "over-adjust." In this case, an advance in the circadian pacemaker would be warranted rather than the large phase delay suggested by Czeisler et al., (1990). The findings from Czeisler et al., (1990) resulted from subjects inexperienced with night work who were shifted to a night active schedule for only five days during the summer months when daylength and the availability of natural light 
exposure are quite different from winter. It is likely that if the Czeisler et al. (1990) protocol were repeated during the winter months with long-term night workers, the experimental manipulations would have effected different results for both groups than those found during the summer.

Clearly then, it is crucial that experiments designed to find treatments to facilitate adaptation to night work be run with actual night workers rather than volunteers who are only shifted to a night schedule for a week or two. The worker's existing circadian phase must be determined and the impact of seasonal variability in the availability of light must be taken into account before considering the appropriate timing of light exposure. For the majority of night workers in the present study, light exposure that would result in a small to moderate phase advance would align the circadian pacemaker with sleep and work. Apparently, at least during the winter months, light exposure in the late afternoon or early evening would result in this corrective phase advance. Therefore, a caveat should be made that the regimen outlined in Czeisler et al. (1990) may not benefit, and might actually create or exacerbate existing problems experienced by night workers who have achieved partial or full adjustment without such treatment. Again, it is crucial that individuals who have been working nights be evaluated with respect to existing circadian phase (as well as possible seasonal variation of circadian phase) prior to beginning treatment.

In addition to the possibility of treating night workers with appropriately timed bright light, there is evidence that properly 
timed administration of oral melatonin will result in shifts in the phase of the circadian pacemaker (Arendt, Aldhous, and Marks, 1986; Sack and Lewy, 1988; Sack, Lewy and Hoban, 1987).

Melatonin is an experimental drug and is not readily available to the public at this time. However, other than mild sedation in some cases, there are no apparent side-effects associated with its use at physiological levels. There is already some interest in marketing melatonin as an aid to combat jet lag. The similarities between jet lag and symptoms experienced by night workers are evident and perhaps in the not too distant future shift workers will take a "shift lag" pill to facilitate adaptation to irregular schedules. It is not yet known if coupling exogenous melatonin administration with appropriately timed exposure to bright light would result in synergistic or additive phase shifting effects. Research in this area could lead to very practical treatments for full-time night workers as well as individuals who are on rotating shifts.

The present study involved expensive and labor intensive procedures which, without greater funding, prohibited recruitment of larger samples. Therefore, data from the present study were necessarily limited and can only be viewed as preliminary. The present investigation was also limited in that recruitment procedures did not make clear the extent to which night workers felt they were "adjusted" or "maladjusted." Although it was explained to subjects that the study was not designed to facilitate adjustment to night work, it is possible that the individuals who responded to the advertisements did so with the hope that they would receive some 
kind of help. Although none of the night workers in the present study reported feeling "very dissatisfied" with their work schedules, there was a great deal of variability among night workers on scores of psychological measures, and it is probable that there was variation within the group in the extent to which individuals felt they had adjusted to their schedules. In the future it would be interesting to assess this variable by investigating differences between night workers who felt well adjusted and those who felt they were not adjusted.

It was originally proposed that lack of synchronization among sleep times, work times, and the daily rise and fall of melatonin production might be associated with a host of both physical and psychological complaints. While the psychological data presented from the present study may be interesting on a case-by-case basis, with the small number of subjects investigated and the high degree of variability, no detailed analyses could be performed.

There has been some support for the idea that morning types do not adapt well to the demands of night work due to "less flexible" circadian rhythms (Ostberg, 1973). It has also been suggested that morning types may be too alert in the morning after the night shift to obtain sufficient amounts of main sleep (Akerstedt, Torsvall and Gillberg, 1989). These finding seem well supported in the case of N8 who had a highly variable sleep pattern which she rated as "poor," but less so in the case N3 who had a fairly consistent sleep pattern and rated his sleep as "excellent." Although very preliminary, the data from these two subjects as well as data from the two 
moderately evening type subjects (N5 and N7) are interesting and suggest that further research investigating the possible relationships among diurnal type, sleep strategy, and the ability to adapt to night work may be fruitful. Unfortunately, the instrument used to assess diurnal type in the present study may have been inadequate. Although the Morningness-Eveningness Questionnaire (Horne and Ostberg, 1976) was validated externally with both self-report measures of perceived alertness and objective measures of oral temperature, little or no information is given on the internal consistency of this scale (Smith, Reilly and Midkiff, 1989). It is also the case that this questionnaire contains items that may be confusing or inappropriate when attempting to assess diurnal type of individuals working irregular shifts.

The results from the Eysenck personality inventory showed that seven of the nine subjects fit the prediction that low neurosis scores would be associated with adequate physiological adjustment to a night work schedule whereas high neurosis score would be associated with difficulties adjusting. Subjects N1, N2 and N3 all scored below or within the normal range on the neuroticism dimension, subjects N6, N8 and N9 scored above the normal range and subject N5 scored at the average. Subject N7 who was not well adjusted (perhaps the least) scored below average on neurosis. In this case it could be argued that stability (the obverse of neurosis), is a necessary, but not sufficient condition for adaptation. Less can be said about subject $\mathrm{N} 4$ who had an elevated neurosis score, yet by the definitions of this investigation was fairly well adapted. 
The results from the POMS were much more variable and no specific pattern of scores was apparent. The POMS was included as a measure in this study because it was thought that the POMS factors (i.e., tension, fatigue, depression, anger, vigor and confusion) would be relevant to the issue of physiological adaptation (or a lack thereof) to night work. Because the POMS is designed to measure changing states of mood rather than durable traits, it may have been more efficacious to use it repeatedly to measure diurnal variability in mood and/or the differences between work days and days away from work. However, the daily ratings of mood that were recorded by subjects showed little variation between groups, little variation between work days and days away from work for the night workers and little diurnal variation for either group.

Although shift work is not a new phenomenon and there was early interest in the question of physiological adaptation, the new generation of research in this area has only begun within the past decade or so. Technologies which allow more reliable monitoring and analyses of circadian rhythms are evolving quickly. This coupled with the relatively recent findings that both sufficiently bright light and exogenous melatonin administration (when appropriately timed), create shifts in the timing of the circadian pacemaker (and by inference, hold or entrain the pacemaker) so that "biological time" is synchronized with the preferred time of work, sleep and leisure offer some long awaited hope.

Yet we must be somewhat cautious with these new findings and keep in mind that the effect of night work on humans is a 
complicated and multidimensional problem. It is doubtful that there is one simple answer that will help the millions who work nights or variable shifts that include nights. It is evident to anyone who has attempted to adjust to night work that in addition to the difficulty of physical adaptation, there are a myriad of psychological and social conflicts experienced on a daily basis. It is critical that further investigations in this area be conducted in field settings with actual shift workers in order for psychosocial factors to be adequately examined. Issues of transient adaptation and seasonal variation need to be addressed. In order for this to occur, future investigations need to be designed in such a way that subjects are evaluated longitudinally, at different times of year.

One thing is certain. We have become a 24-hour society. As the need increases for business and services to run on a 24-hour basis, the division between night and day becomes less clear for growing numbers of individuals. Many of these individuals are in professions that require high levels of alertness and performance. It is important that we remember that the phenomenon of shift work effects not only individuals working unconventional hours; it effects the safety and well-being of us all. 


\section{REFERENCES}

Ackerstedt, T., Froberg, J.E., Friberg, Y. \& Wetterberg, L. (1979). Melatonin secretion, body temperature and subjective arousal during 64 hours of sleep deprivation. Psychoneuroendocrinology, 4, 219-225.

Ackerstedt, T., Torsvall, L. \& Gillberg, M. (1989). Shiftwork and napping. In D.F. Dinges and R.J. Broughton (Eds.), Sleep and Alertness: Chronobiological, behavioral, and medical aspects of napping (pp. 205-220). New York: Raven Press.

Arendt, J. (1979). Radioimmunoassayable melatonin: Circulating patterns in man and sheep. In J. Ariens Kappers and P. Pevet (Eds.), The pineal gland of vertebrates including man, progress in brain research vol. 52. (pp. 249-258). North Holland, New York: Elsivier.

Arendt, J. (1981). Current status of assay methods of melatonin. In N. Birau \& W. Schoot (Eds.), Melatonin - Current status and perspectives. Advances in the biosciences (pp. 3-7). New York: Pergamon Press.

Arendt, J., Aldhous, M. \& Marks, V. (1986). Alleviation of jet lag by melatonin: Preliminary results of controlled double blind trial. British Medical Journal, 292, 1170.

Aschoff, J. (1979). Circadian rhythms: General features and endocrinological aspects. In D.T. Kreiger, (Ed.), The pineal gland (pp. 77-128). North-Holland, New York: Elsevier.

Blake, M.J.F. (1967). Relationship between circadian rhythms of body temperature and introversion-extraversion. Nature, 21(5), 896-897. 
Boulos, Z., Rossenwasser, A.M., \& Terman, M., (1980). Feeding schedules and the circadian organization of behavior in the rat. Behavioral Brain Research, 1,39-65.

Campbell, S., Dawson, D., Eastman, C.I \& Lewy, A.J. (1990). Light treatment for shift work. Light Treatment and Biological Rhythms. Bulletin of the Society for Light and Biological Rhythms,. 2 (5), 9-10.

Colquhoun, W.P. (1960). Temperament, inspection efficiency, and time of day. Ergonomics, 3, 377-378.

Colquhoun, W.P., Blake, M.J.F. and Edwards, R.S. (1968a).

Experimental studies of shift-work II: Stabilized 8-hour shift systems. Ergonomics, 11(6), 527-546.

Colquhoun, W.P., Blake, M.J.F. and Edwards, R.S. (1968b).

Experimental studies of shift-work III: Stabilized 12-hour shift systems. Ergonomics, 12(6), 865-882.

Colquhoun, W.P. \& Corcoran, D.W.J. (1964). The effect of time of day and social isolation on the relationship between temperament and performance. British Journal of Social Clinical Psychology, 3, 266-231.

Czeisler, C.A. (1982). Rotating shift work schedules that disrupt sleep are improved by applying circadian principles. Science, 217, 460-463.

Czeisler, C.A., Brown, E.N., Ronda, J.M., Kronauer, R.E., Richardson, G.S. \& Freitag, W.O. (1985). A clinical method to assess the endogenous circadian phase (ECP) of the deep circadian oscillator in man. Sleep Research, 14, 295.

Czeisler, C.A., Johnson, M.P., Duffy, J.F., Brown, E.N., Ronda, J.M. \& Kronauer, R.E. (1990). Exposure to bright light and darkness to treat physiologic maladaptation to night work. The New England Journal of Medicine, 322(18), 1253-1259. 
Czeisler, C.A., Richardson, G.S., Zimmerman, J.C., Moore-Ede, M.C.\&Weitzman, E.D. (1981). Entrainment of human circadian rhythms by light-dark cycles: A reassessment. Photochemistry and Photobiology, 34, 239-247.

Czeisler, C.A., Kronauer, R.E., Allen, J.S., Duffy, J.F., Jewett, M.E. Brown, E.N. \& Ronda, J.M. (1989). Bright light induction of strong (Type 0 ) resetting of the human circadian pacemaker. Science, 244, 1328-1332.

Czeisler, C.A., Weitzman, E.D., Moore-Ede, M.C. Zimmerman, J.C. and Knauer, R.S. (1980). Human sleep: Its duration and organization depend on its circadian phase. Science, 210, 1264-1267.

Eysenck, H.J. and Eysenck, B.G. (1968). The Manual for the Eysenck Personality Inventory. Educational and Industrial Testing Service, (pp. 5-28).

Folkard, S., Minors, D.S. \& Waterhouse, J.M. (1985). Chronobiology and shiftwork: current trends and issues. Chronobiologia, 12 . 31-54.

Folkard, S., Monk, T. \& Lobban, M. (1978). Short and long term adjustment of circadian rhythms in "permanent" night nurses. Ergonomics, 21, 785-799.

Gorden, N.P., Cleary, P.D., Parker, C.E. \& Czeisler, C.A. (1986). The prevalence and health impact of shiftwork. American Journal of Public Health, 76(10), 1225-1228.

Herbert, D.C. \& Reiter, R.J. (1981). Influence of protein-calorie malnutrition on the circadian rhythm of melatonin in the rat. Proceedings of the Society for Experimental Biology and Medicine, $166,360-363$.

Horne, J.A. (1985). Sleep loss: Underlying mechanisms and tiredness. In S. Folkard, \& T.H. Monk (Eds.), Hours of work: Temporal factors in work-scheduling (pp. 53-65).

New York: John Wiley \& Sons. 
Horne, J.A. \& Ostberg, O. (1976). A self-assessment questionnaire to determine morningness-eveningness in human circadian rhythms. International Journal of Chronobiology, 4. 97-110.

Illnerova, H. (1976). The effects of immobilization on the activity of serotonin $\mathrm{N}$-acetyltransferase in the rat epiphysis. In $\mathrm{E}$. Usdin, R. Ketnanasky and I.J. Kopin (Eds.), Catecholamines and stress (pp. 129-136). New York: Pergamon Press.

Inouye, S.T. \& Sawamura, H. (1977). Characteristics of a circadian pacemaker in the suprachiasmatic nucleus. Journal of Comparative Physiology, 146, 153-160.

Jimmerson, D.C., Lynch, H.J., Post, R.M., Wurtman, R.J. \& Bunney, W.E. (1977). Urinary melatonin rhythms during sleep deprivation in depressed patients and normals. Life Sciences, 20, 1501-1508.

Johnson, L.C., Tepas, D.I., Colquhoun, W.J. \& Colligan, M.J. (Eds.), (1981). Preface to The Twenty-four Hour Workday: Proceedings of a Symposium on Variations in Work-Sleep Schedules. DHHS Pub. No. 81-127 (NIOSH). Washington, DC: Govt. Printing Office.

Klein, D.C., \& Parfitt, A. (1976). A protective role of nerve endings in stress-stimulated increase in the pineal $\mathrm{N}$-acetyltransferase activity. In E. Usdin, R. Ketnanasky and I.J. Kopin (Eds.), Catecholamines and stress (pp. 129-136). New York: Pergamon Press.

Knauth, P., Rutenfranz, J., Herrmann, G. \& Poppel, S.J. (1978). Reentrainment of body temperature in experimental shift work studies. Ergonomics, 21, 775-783.

Knauth, P., Rutenfranz, J., Shultz, H., Bruder, S., Romberg, H.P, Decoster, F.\& Kiesswetter, E. (1980). Experimental shift work studies of permanent night, and rapidly-rotating, shift systems. II. Behavior of various characteristics of sleep. International Archives of Occupational and Environmental Health, 46, 111-125.

Lewy, A.J. (1983). Biochemistry and regulation of mammalian melatonin production. In R.M. Relkin, (Ed.), The pineal gland (pp. 77-128). North-Holland, New York: Elsevier. 
Lewy, A.J. \& Markey, S.P. (1978). Analysis of melatonin in human plasma by gas chromatography-negative chemical ionization mass spectrometry. Science, 201. 741-743.

Lewy, A.J., Sack, R.L. \& Singer, C.M. (1984). Assessment and treatment of chronobiologic disorders using plasma melatonin levels and bright light exposure: The clock-gate model and phase response curve. Psychopharmacology Bulletin, 20(3), $561-565$.

Lewy, A.J., Wehr, T.A., Goodwin, F.K., Newsome, D.A. and Markey, S.P. (1980). Light suppresses melatonin secretion in humans. Science, 210, 1267-1269.

Lund, R. (1974). Personality factors and desynchronization of circadian rhythms. Psychosomatic Medicine, 36, 224-228.

Lynch, H.J. (1983). Assay methodology. In R.M.Relkin, (Ed.), The pineal gland (pp. 129-146). North Holland-New York: Elsevier.

Lynch, H.J., Eng, J.P. \& Wurtman, R.J. (1973). Control of pineal indole biosynthesis by changes in sympathetic tone caused factors other than environmental lighting. Proceedings of the National Academy of Sciences. USA, 70, 1704-1708.

McNair, D.M. Lorr, M. \& Droppleman, L.F. (1971). The Manual for the Profile of Mood States. Educational and Industrial Testing Service, (pp. 5-29).

Mills, J.N., Minors, D.S., and Waterhouse, J.M. (1978a). Adaptation to abrupt shifts of the oscillators controlling human circadian rhythms. Journal of Physiology, 285, 455-470.

Mills, J.N., Minors, D.S., and Waterhouse, J.M. (1978b). Exogenous and endogenous influences on rhythms after sudden time shift. Ergonomics, 21(10), 755-761. 
Mills, J.N., Minors, D.S. \& Waterhouse, J.M. (1974). The circadian rhythms of human subjects without timepieces or indication of the alteration of day and night. Journal of Physiology, 240, 567594.

Minors, D.S. \& Waterhouse, J.M. (1981). Circadian rhythms and the human. Bristol: Wright-PSG.

Minors, D.S. \& Waterhouse, J.M. (1988). Effects upon circadian rhythmicity of an alteration to the sleep-wake cycle: Problems of assessment resulting from measurement in the presence of sleep and analysis in terms of a single shifted component. Journal of Biological Rhythms, 3(1), 23-40.

Moog, R. and Hildebrandt, G. (1986). Comparisons of different causes of masking effects. In M. Haider, M. Koller and R. Cervinka (Eds.), Night and shiftwork: Long-term effects and their prevention (pp. 131-140). Peter Lang Verlag: Frankfurt.

Moore, R.Y. \& Klein, D.C. (1974). Visual pathways and the central neural control of a circadian rhythm in pineal serotonin $\mathrm{N}$ acetyltransferase activity. Brain Research, 71, 17-33.

Moore-Ede, M.C., Sulzman, F.M., Fuller, C.A. (1982). The clocks that time us: Physiology of the human circadian timing system Cambridge: Harvard University Press.

Moore-Ede, M.C. \& Richardson, G.S. (1985). Medical implications of shift-work. Annual Review of Medicine, 36, 607-617.

Ostberg, O. (1973). Interindividual differences in circadian fatigue patterns of shift workers. British Journal of Industrial Medicine, 30, 341-351.

Rollag, M.D. (1981). Methods for measuring pineal hormones. In R.J. Reiter, (Ed.), The pineal gland. Anatomy and biochemistry Vol. 1, (pp. 273-302). Boca Raton, Florida: CRC Press.

Rutenfranz, J. \& Knauth, P. (1988). Shift work. In Carl Zenz, (Ed.), Occupational medicine: Principles and practical applications Chicago: Year Book Medical Publishers, Inc. 
Rutenfranz, J. \& Knauth, P. and Colquhoun, W.P. (1976). Hours of work and shiftwork. Ergonomics, 19(3), 331-340.

Sack, R.L. \& Lewy, A.J. (1988). Melatonin administration phase advances endogenous rhythms in man. Sleep Research, 17, 396.

Sack, R.L., Lewy, A.J. and Hoban, T.M. (1987). Free running melatonin rhythm in blind people: Phase shifts with melatonin and triazolam administration. In $U$. Rensing, $U$. an der Heiden and M.C. Makey (Eds.), Temporal disorder in human oscillatory systems (pp. 219-224). Heidelberg: Springer-Verlag.

Shiftwork Committee (1979). Japan association of Industrial Health. Opinion on night work and shift work. Journal of the Science of Labour, Part II, 1-36.

Smith, C.S., Reilly, C. \& Midkiff, K. (1989). Evaluation of three circadian rhythm questionnaires with suggestions for an improved measure of morningness. Journal of Applied Psychology, 74 (5), 728-738.

Tasto, D.L., Colligan, M.J., Skejei, E.W., \& Polly, S.J. (1978). Health consequences of shift work. (pp. 88-134). NIOSH technical report No. 78-154. Washington, DC: Govt. Printing Office.

Tepas, D.I., Walsh, J.K, \& Armstrong, D. (1981). Comprehensive study of the sleep of shift workers. In L.C.Johnson, W.P. Tepas \& M.J. Colligan, (Eds.), The Twenty-Four Hour Workday: Proceedings of a Symposium on Variations in Work-Sleep Schedules (pp. 419-433). DHHS Pub. No. (NIOSH). Washington, DC: Govt. Printing Office.

Van Cauter, E. \& Turek, F.W. (1990). Strategies for resetting the human circadian clock. New England Journal of Medicine. 322, 1306-1308.

Van Loon, J.H. (1963). Diurnal body temperature curves in shift workers. Ergonomics, 6, 267-273. 
Vaughan, G.M., Bell, R. \& De La Pena, A. (1979). Nocturnal plasma melatonin in humans: Episodic pattern and influence of light. Neuroscience Letters, 14, 81-84.

Vaughan, G.M., McDonald, S.D., Jordan, R.M., Allen, J.P., Bohmfalk, G.L., Abou Samra, M. \& Story, J.L. (1978). Melatonin concentration in human blood and cerebrospinal fluid: Relationship to stress. Journal of Clinical Endocrinology and Metabolism, 47, 220-223.

Waldhauser, F., Vierhapper, H., \& Pirich, K. (1986). Abnormal circadian melatonin secretion in night-shift workers. New England Journal of Medicine, 1614.

Weitzman, E.D., Czeisler, C.A., \& Moore-Ede, M.C. (1979). Sleep-wake, neuroendocrine and body temperature circadian rhythms under entrained and non-entrained (free-running) conditions in man. In M. Suda, O. Hayaishi \& H. Nakagawa, (Eds.), Biological rhythms and their central mechanisms (pp. 199-227). North Holland, New York.: Elsevier

Weitzman, E.D., \& Kripke, D.F. (1981). Experimental 12-hour shifts of the sleep-wake cycle in man: Effects on sleep and physiological rhythms. In L.C. Johnson, D.I. Tepas, W.P.Colquhoun\& M.J.Colligan, (Eds.), Advances in sleep research, vol. 7, biological rhythms. sleep and shift work, (pp. 93-110). New-York: Spectrum Publications.

Wever, R.A. (1979). The circadian system of man. Results of experiments under temporal isolation (pp. 25-124) New York: Springer Verlag.

Wever, R.A. (1989). Light effects on human circadian rhythms; A review of recent Andechs experiments. Journal of Biological Rhythms, 4, 161-184.

Zulley, J. Wever, R., \& Aschoff, J. (1981). The dependence of onset and duration of sleep on the circadian rhythm of rectal temperature. Pflugers Archives, 391, 314-318. 
APPENDIX A

DAILY DIARY AND

INSTRUCTIONS 


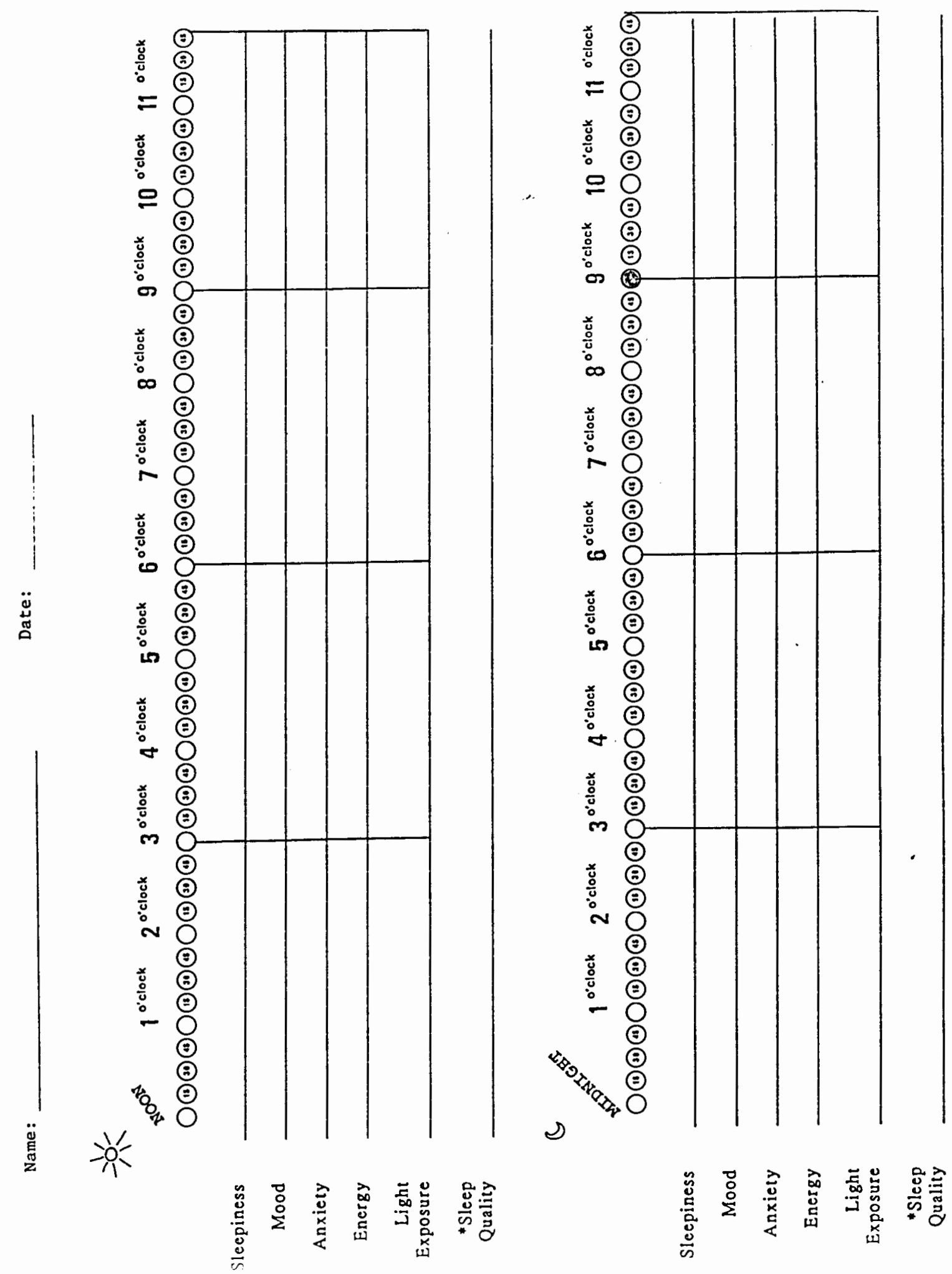




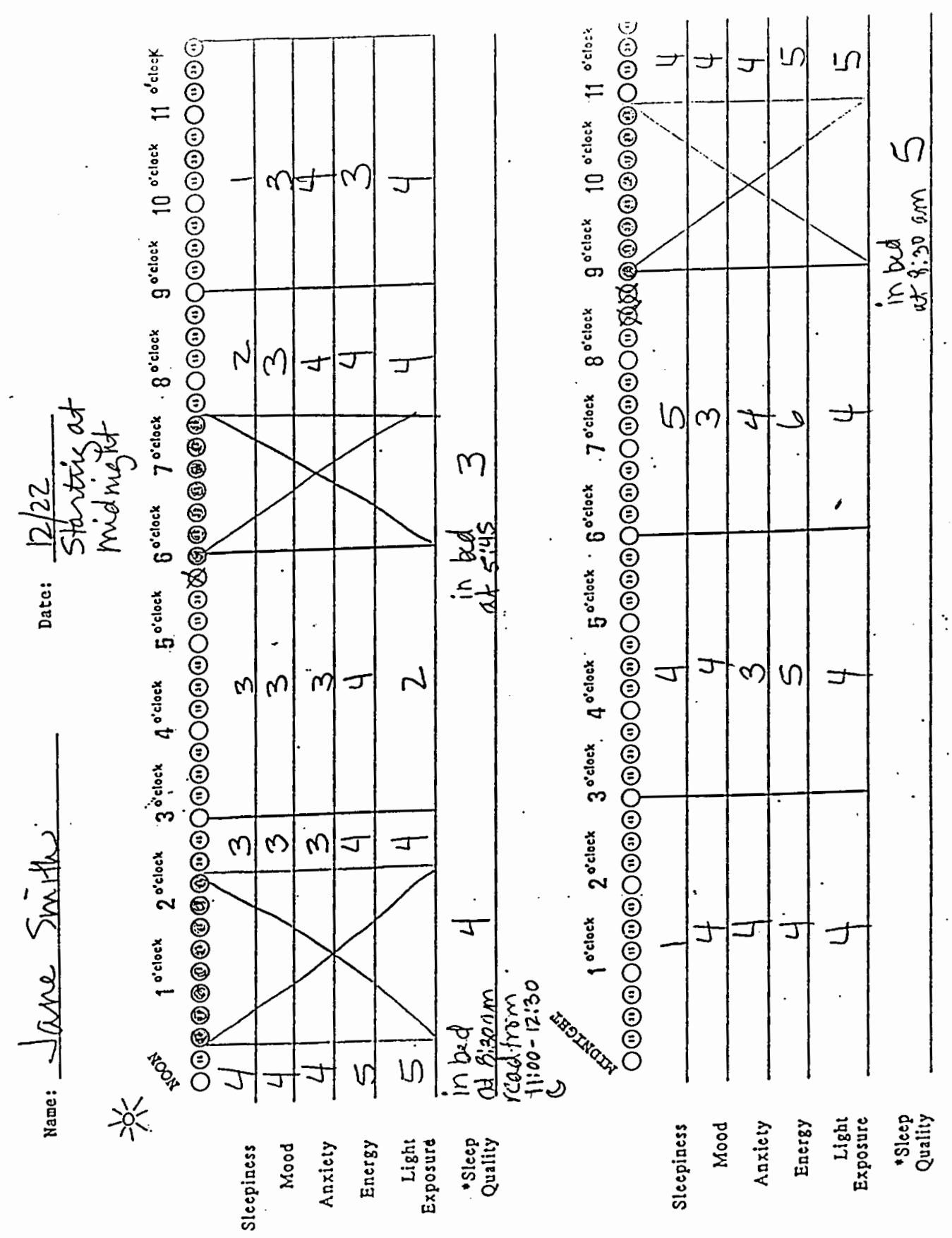


Instructions for Rating Form

1. Start form at midnight (bottom section) every day. You do not have to do the ratings hour by hour but try to make the ratings as the day (night) progresses. It is not a good idea to wait longer than 24-hours.

2. Awake: For times when you are awake, use the attached rating form and scale to rate sleepiness, mood, anxiety, energy level and light exposure. You need only make one rating for each section. (i.e., rate once for time period between vertical lines)

3. Sleep: Mark the corresponding time "bubble" with an $X$ when you lay down to sleep. Blacken all bubbles for the times that you estimate that you were asleep (see example). For the times you are asleep rate sleep quality only using the attached sleep quality rating scale. 
Name:

RATING SCALES

\begin{tabular}{|c|c|c|c|c|c|}
\hline Score & Sleepiness & Mood & Anxiety & Energy & Ilght Exposure \\
\hline 1 & $\begin{array}{l}\text { Feellng active and vital; } \\
\text { alert; wide awake. }\end{array}$ & $\begin{array}{l}\text { Very } \\
\text { happy }\end{array}$ & $\begin{array}{l}\text { Very } \\
\text { relaxed }\end{array}$ & $\begin{array}{l}\text { Very } \\
\text { energetic }\end{array}$ & $\begin{array}{l}\text { Bright } \\
\text { outdoor }\end{array}$ \\
\hline 2 & $\begin{array}{l}\text { Functioning at a high } \\
\text { level but not at peak } \\
\text { able to concentrate. }\end{array}$ & $\begin{array}{l}\text { Quite } \\
\text { happy }\end{array}$ & $\begin{array}{l}\text { Quite } \\
\text { relaxed }\end{array}$ & $\begin{array}{l}\text { Quite } \\
\text { energetic }\end{array}$ & $\begin{array}{l}\text { Dim } \\
\text { outdoor } \\
\text { (cloudy) }\end{array}$ \\
\hline 3 & $\begin{array}{l}\text { Relaxed; awake; not at } \\
\text { full aleztness; responsive. }\end{array}$ & $\begin{array}{l}\text { Tend to } \\
\text { be happy }\end{array}$ & $\begin{array}{l}\text { Tend to } \\
\text { be relaxed }\end{array}$ & $\begin{array}{l}\text { Above average } \\
\text { energy level }\end{array}$ & $\begin{array}{l}\text { Bright } \\
\text { interior }\end{array}$ \\
\hline 4 & $\begin{array}{l}\text { A little foggy: not at } \\
\text { peak; let down. }\end{array}$ & Neutral & Not anxious & $\begin{array}{l}\text { Average } \\
\text { energy }\end{array}$ & $\begin{array}{l}\text { Usual } \\
\text { Intertor }\end{array}$ \\
\hline 5 & $\begin{array}{l}\text { Fogglness; beginning to } \\
\text { lose interest in remaining } \\
\text { awake; slowed down. }\end{array}$ & $\begin{array}{l}\text { Tend to } \\
\text { be sad }\end{array}$ & $\begin{array}{l}\text { Tend to be } \\
\text { anxious }\end{array}$ & $\begin{array}{l}\text { Below average } \\
\text { energy level }\end{array}$ & $\begin{array}{l}\text { Dim } \\
\text { Interior }\end{array}$ \\
\hline 6 & $\begin{array}{l}\text { Sleepiness; prefer to be } \\
\text { lying down; flghting } \\
\text { sleep; woozy. }\end{array}$ & $\begin{array}{l}\text { Quite } \\
\text { sad }\end{array}$ & $\begin{array}{l}\text { Quite } \\
\text { anxious }\end{array}$ & $\begin{array}{l}\text { Quite } \\
\text { tired }\end{array}$ & $\begin{array}{l}\text { Dark } \\
\text { Interlor } \\
\text { (candlellght) }\end{array}$ \\
\hline 7 & $\begin{array}{l}\text { Almost in reverle; sleep; } \\
\text { onset soon; lost struggle } \\
\text { to remaln awake. }\end{array}$ & $\begin{array}{l}\text { Very } \\
\text { sad }\end{array}$ & $\begin{array}{l}\text { Very } \\
\text { anxious }\end{array}$ & $\begin{array}{l}\text { Very } \\
\text { tired }\end{array}$ & Dark outdoor \\
\hline
\end{tabular}

\section{* Scale for sleep quality ratings:}

$1=$ Very Good; no interruption (sound sleep)

$2=$ Good; awakened a few times but overall slept well.

3 = Fair, light sleep; not satisfactory.

$4=$ Poor: restless; frequent intemuptions and dozing.

$5=$ Bad; very restless; little sleep. 


\section{APPENDIX B}

GRAPHIC REPRESENTATIONS OF

INDIVIDUAL NIGHT WORKER'S

SLEEP PATTERNS 


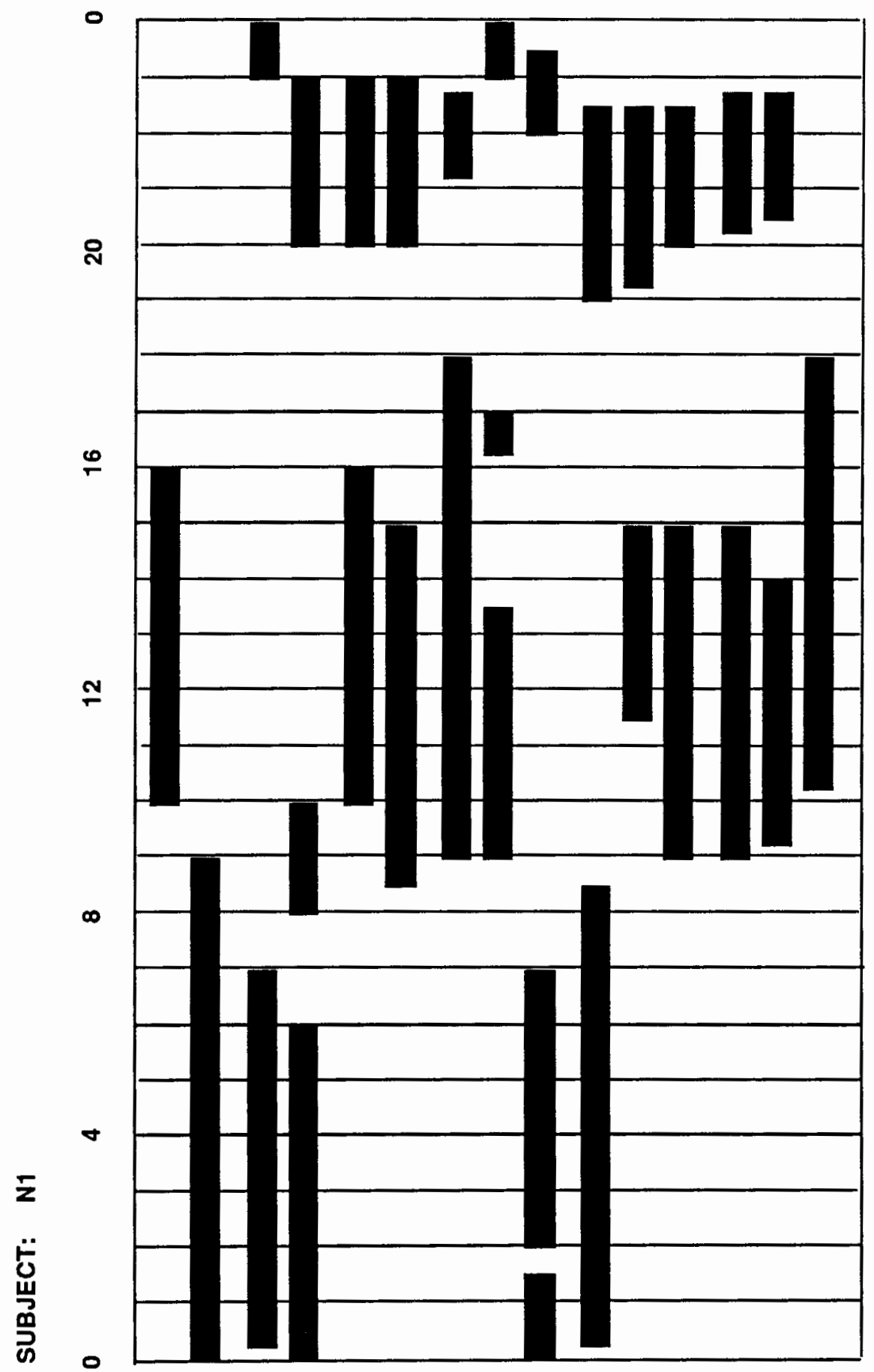




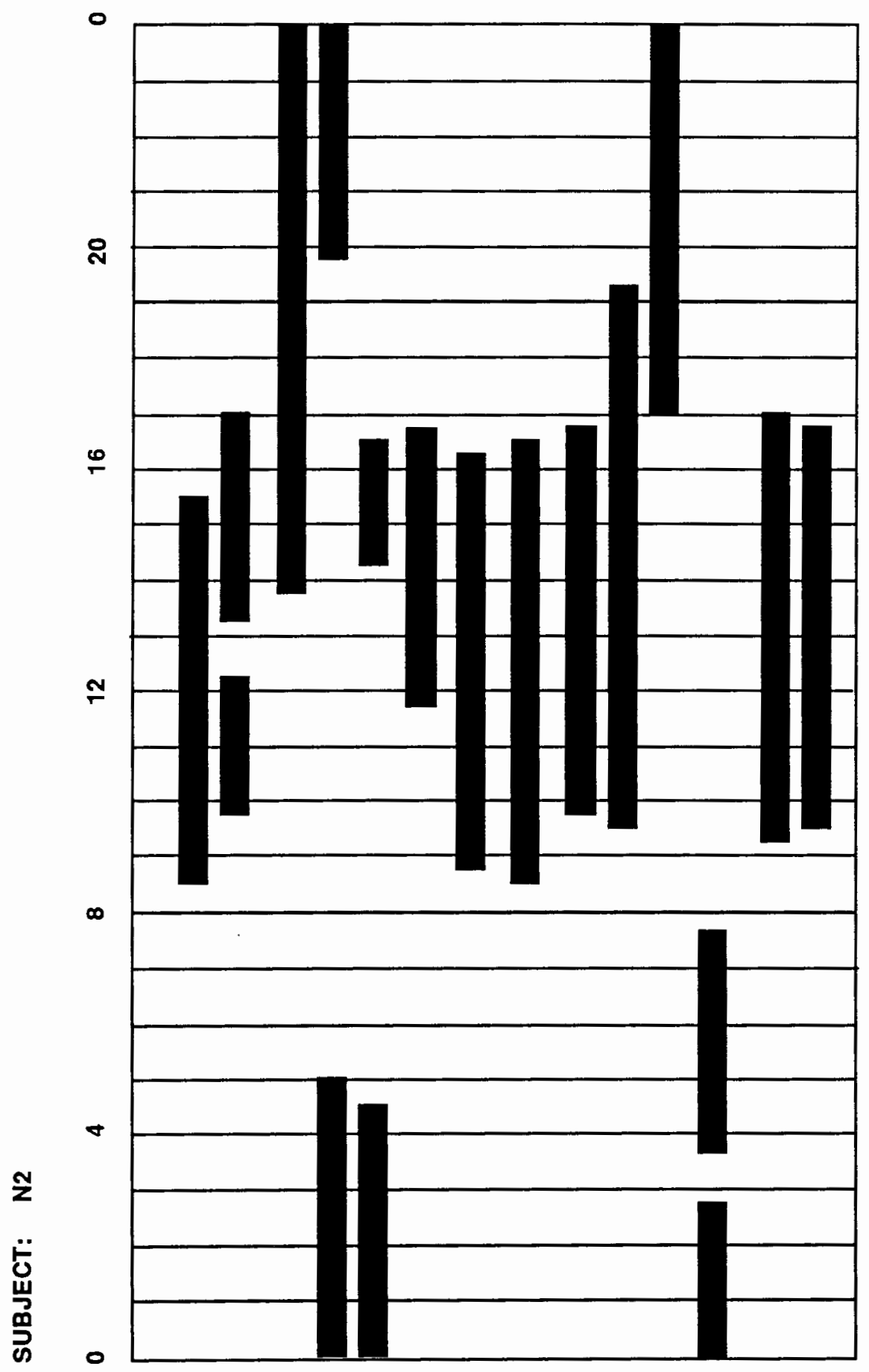




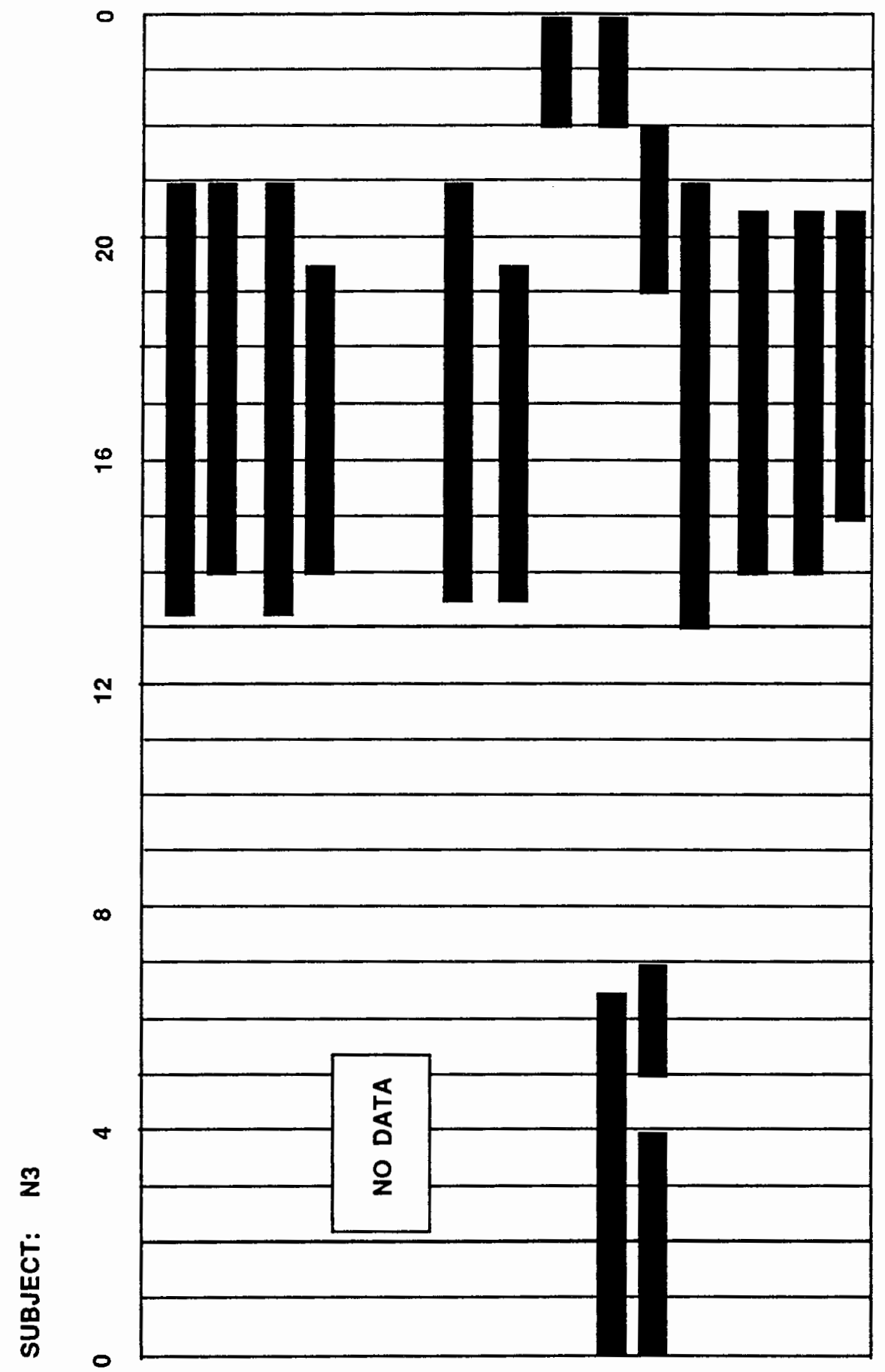




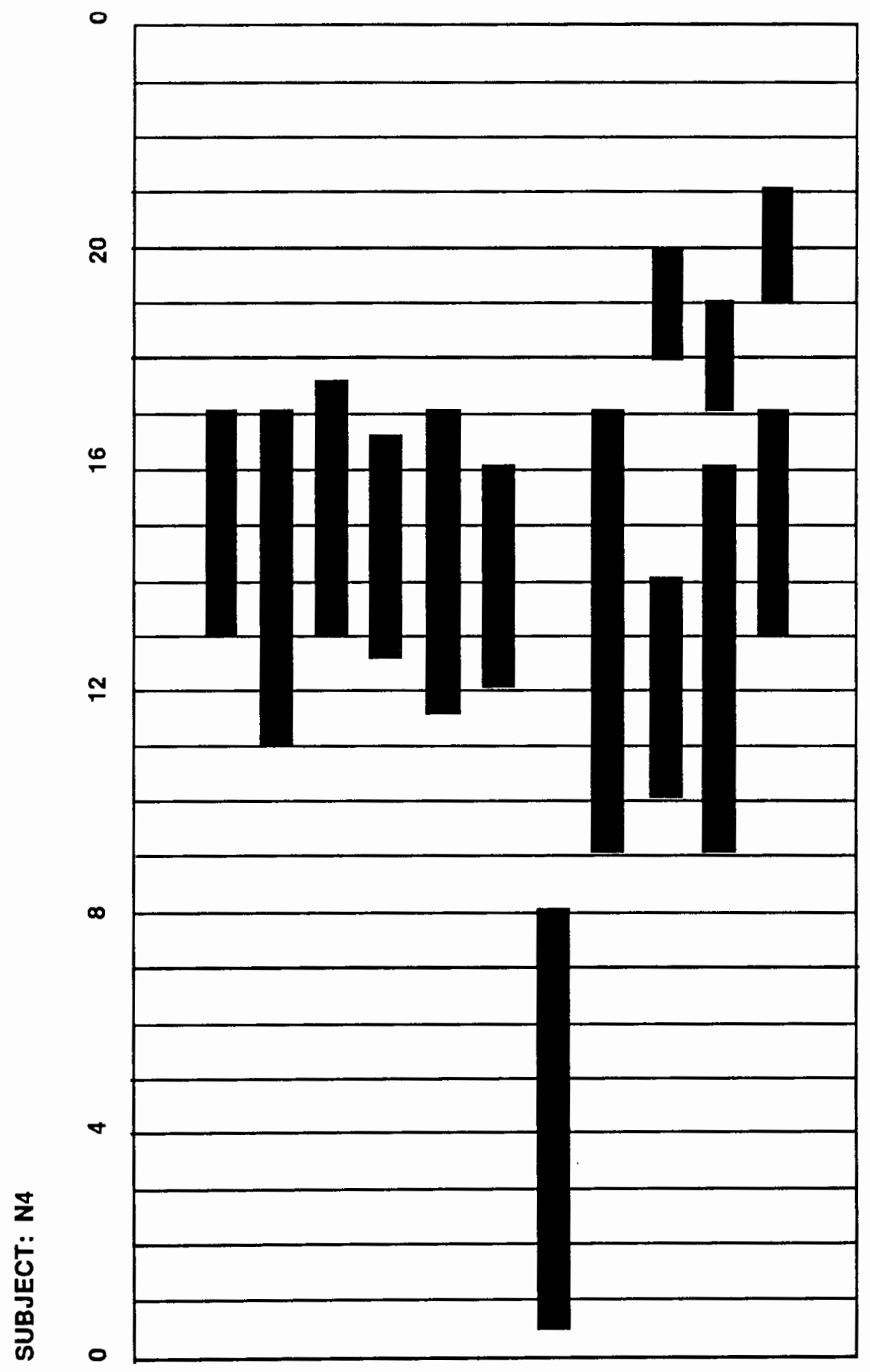




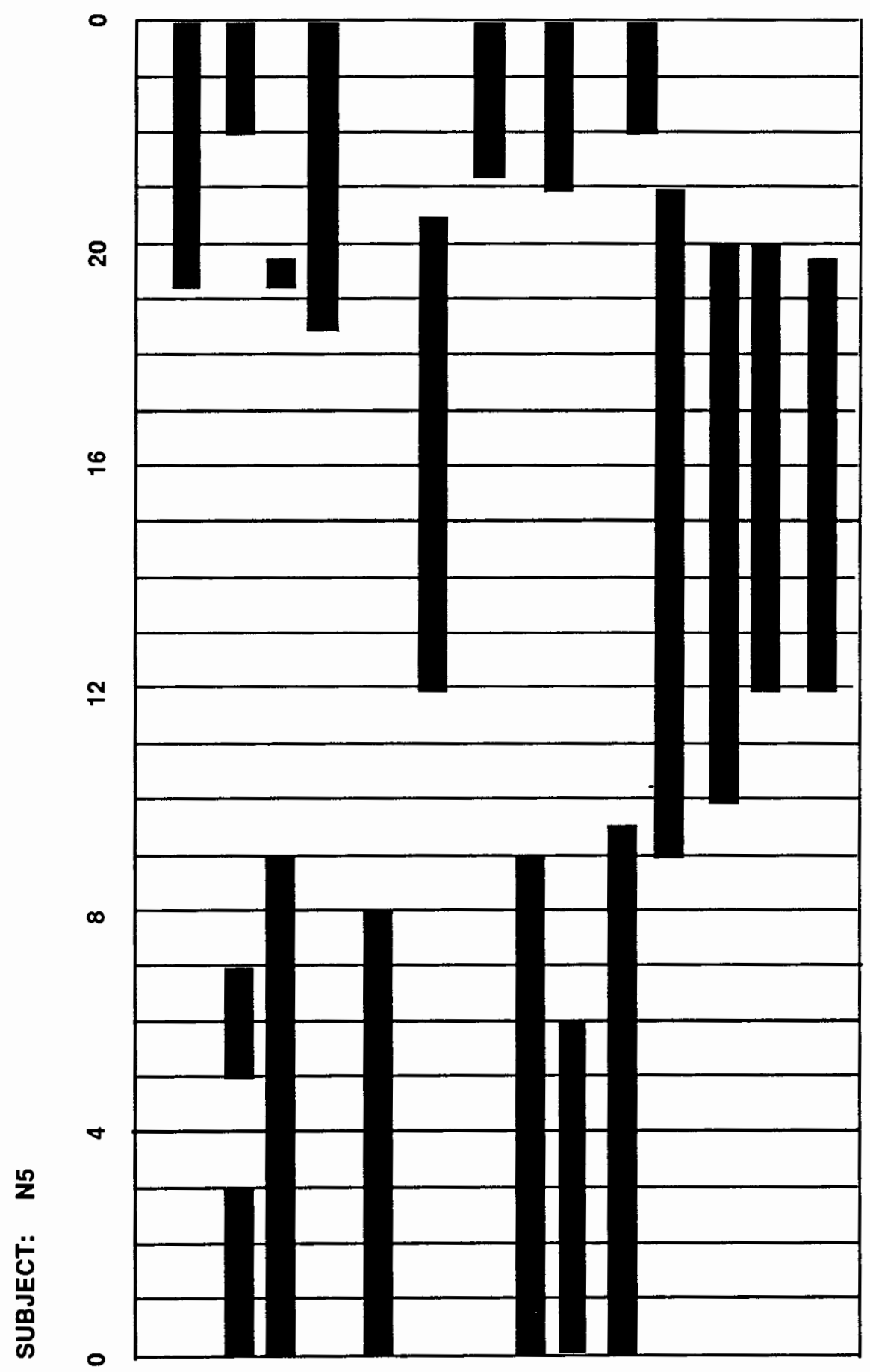




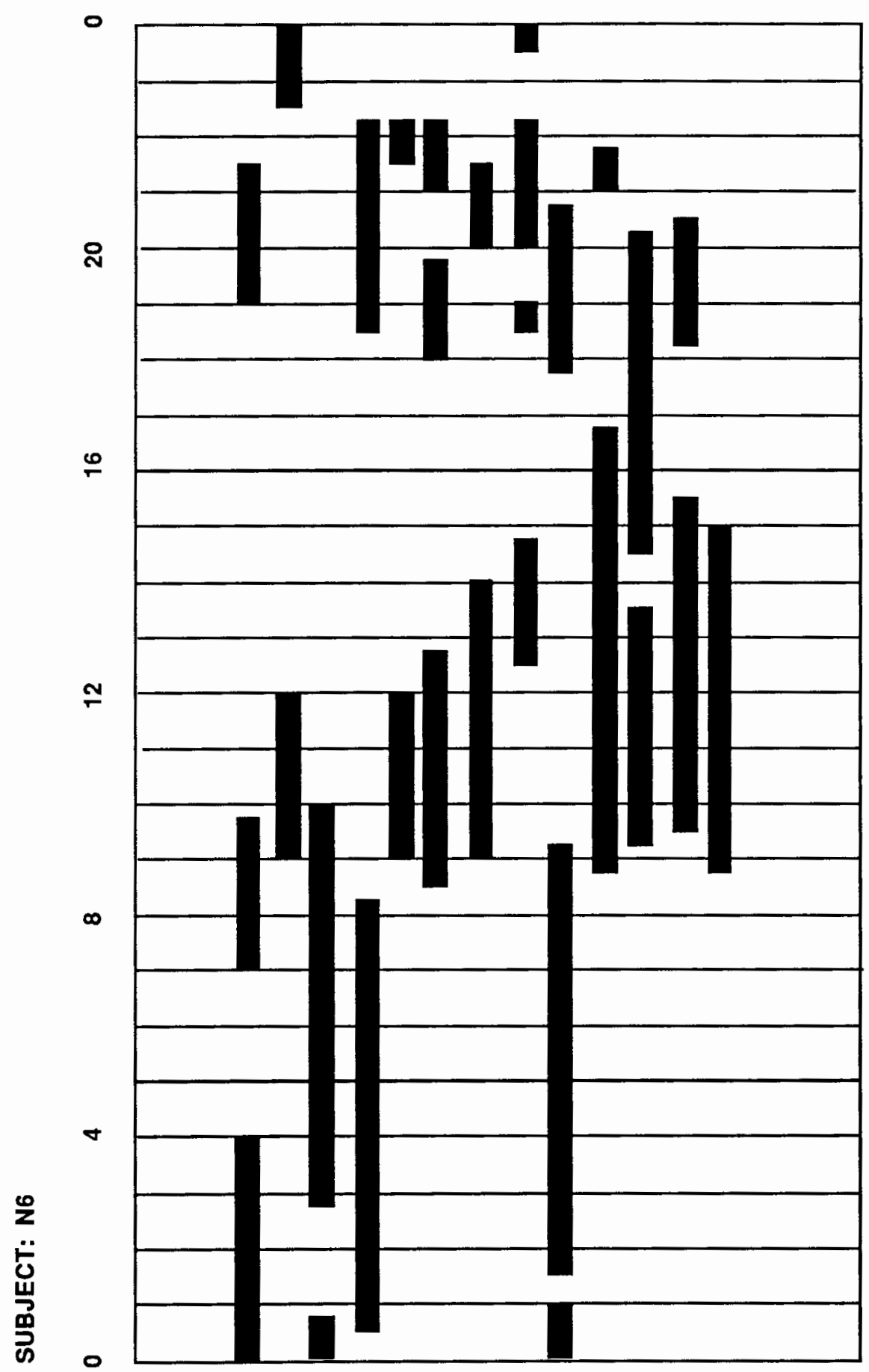




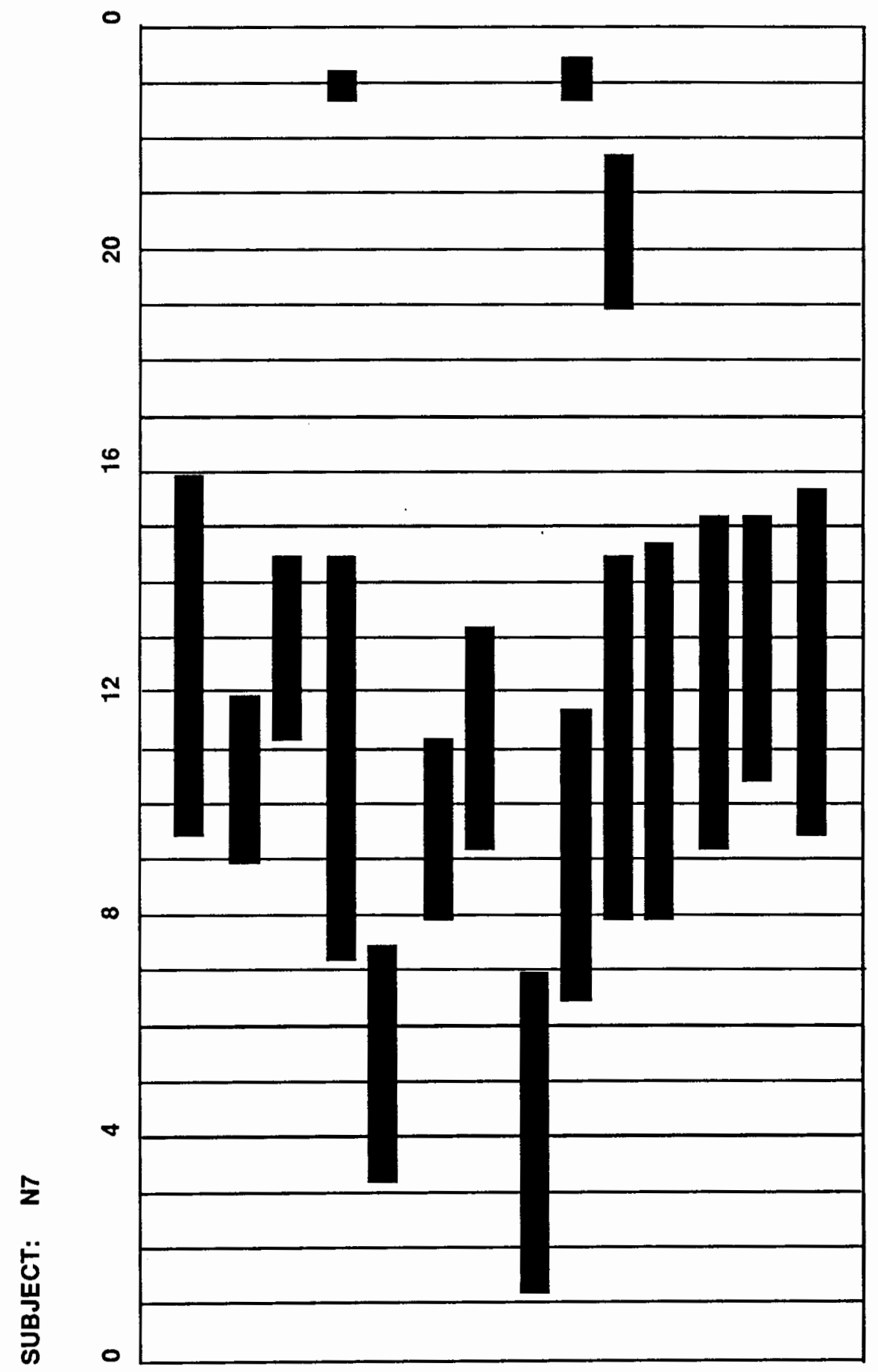




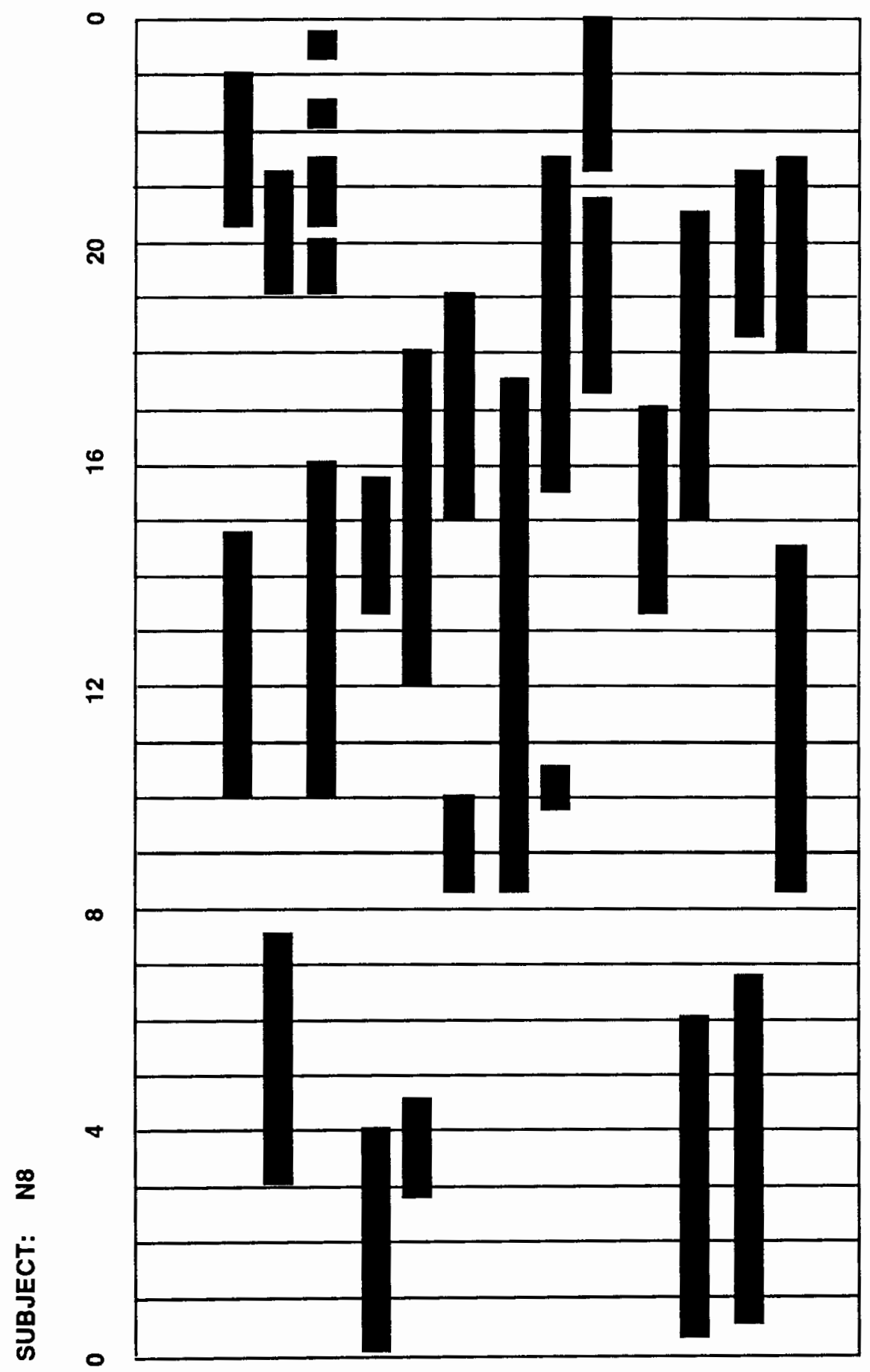




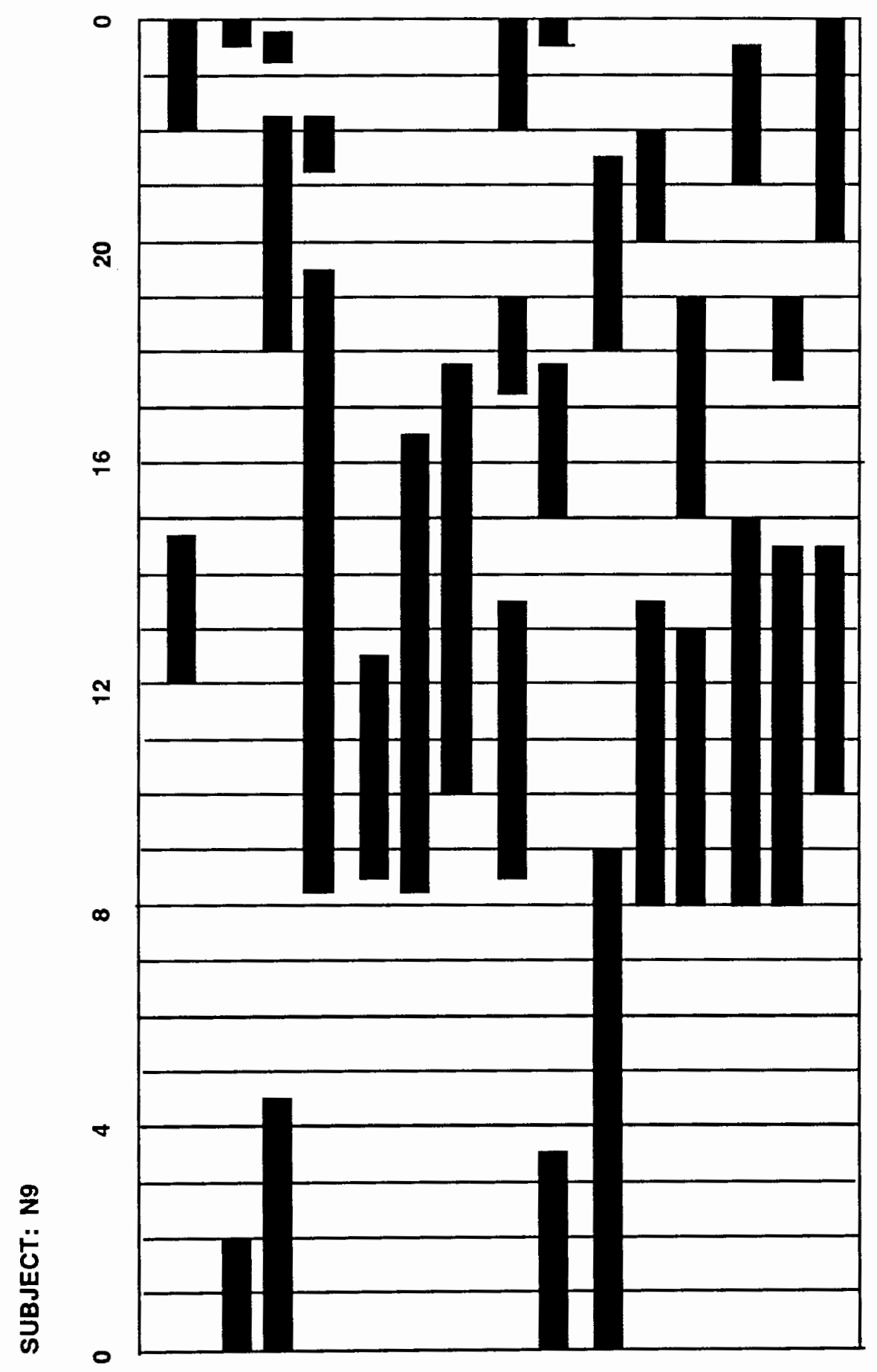




\section{APPENDIX C}

\section{PHYSICAL SYMPTOMS}




\section{PHYSICAL SYMPTOMS}

The Follwing questions concern your body functions. Please try to answer each question by circling a number to indicate how often you have experienced each of the following items within the past year.

Shortness of breath

Frequent colds or sore throat

Persistent cough

Coughing up blood

Fever, chills and aching all over

Hay fever or sinus trouble

Wheezing in your chest

Jaundice, yellow skin or eyes

Itching, rash or allergic skin

Swollen or painful muscles and joints

Back pain

Pain or stiffness in arms or legs

Tearing or itching eyes

Persistent numbness or tingling

Ringing or buzzing in ears

Severe headaches

Fainting spells or dizziness

Nervous or shaking inside

Sweaty or trembly

Increased urination

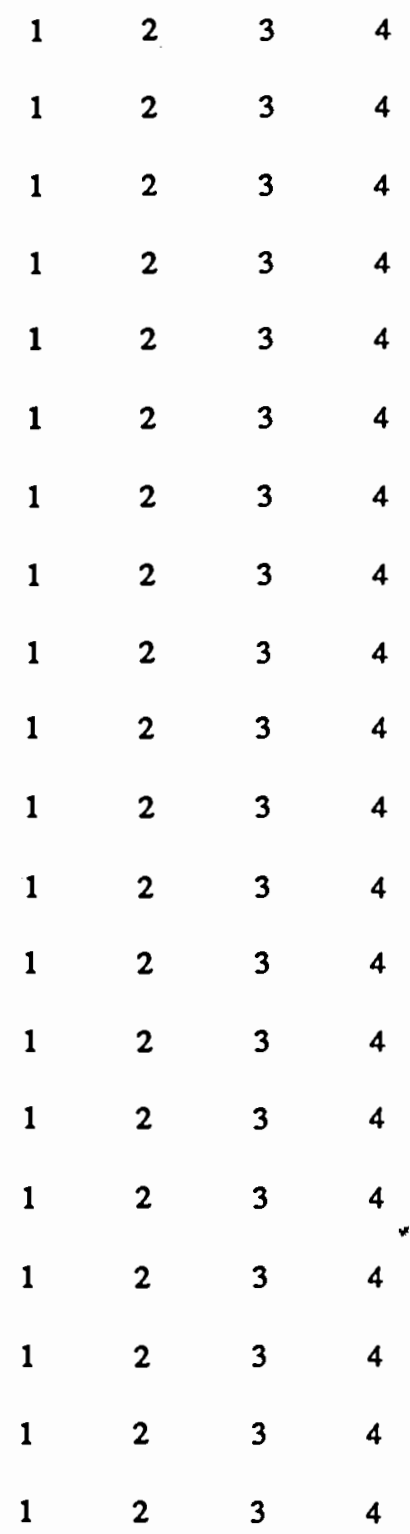


Physical Symptoms Continued

Painful Urination

Bloody Urine

Alarming pain or pressure in chest

Pain down your arms

"Racing" or pounding heart

Leg cramps

Severe fatigue or exhaustion

Acid indigestionor heart burn

Diarrhea for more than a few days

Gas or gas pains

Nausea or vomiting

Blood in bowel movement

Constipation

Tight feeling in stomach

Bloated or full feeling

Feeling pressure in neck

Hemorrhoids or piles

Trouble digesting food

Blurred vision

Dryness in the mouth

Stomach pains

Belching

Difficulty standing for long periods
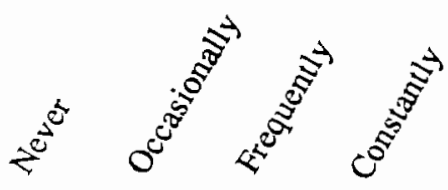

$\begin{array}{llll}1 & 2 & 3 & 4\end{array}$

$\begin{array}{llll}1 & 2 & 3 & 4\end{array}$

$\begin{array}{llll}1 & 2 & 3 & 4\end{array}$

$\begin{array}{llll}1 & 2 & 3 & 4\end{array}$

$\begin{array}{llll}1 & 2 & 3 & 4\end{array}$

$\begin{array}{llll}1 & 2 & 3 & 4\end{array}$

$\begin{array}{llll}1 & 2 & 3 & 4\end{array}$

$\begin{array}{llll}1 & 2 & 3 & 4\end{array}$

$\begin{array}{llll}1 & 2 & 3 & 4\end{array}$

$\begin{array}{llll}1 & 2 & 3 & 4\end{array}$

$\begin{array}{llll}1 & 2 & 3 & 4\end{array}$

$1 \quad 2 \quad 3 \quad 4$

$\begin{array}{llll}1 & 2 & 3 & 4\end{array}$

$\begin{array}{llll}1 & 2 & 3 & 4\end{array}$

$\begin{array}{llll}1 & 2 & 3 & 4\end{array}$


APPENDIX D

LIFESTYLE 


\section{QUESTIONS RELATING TO LIFE SATISFACTION}

Using the following scale, rate how satisfied are you with the amount of time your work schedule allows you to:

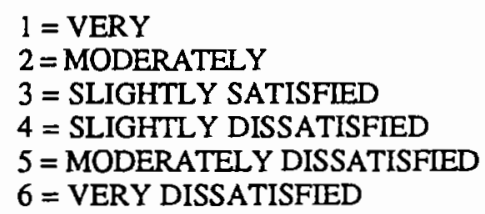

1. Engage in hobbies or fix things

2. Run errands such as going to the bank, hardware store, or barber or beautician?

3. Attend school or training classes

4. Attend meetings

5. Watch T.V. at home

6. Listen to music

7. Read

8. Attend social events

9. Participate in group sports

10. Participate in membership organizations

11. Spend holidays/days off with relatives \& friends

12. In general, how do you feel about your work hours or work schedule?

13. In general, how does your family (or the people you live with) feel about your work hours or work schedule?

14. How do you feel about kind of work you do at your job? 
15. How many of your friends work the same kind of schedule as yourself?

$1=\mathrm{ALL}$

$2=$ MOST

$3=$ SOME

$4=$ NONE

16. How many of your neighbors work the same kind of schedule as yourself?

$1=$ ALL

$2=$ MOST

$3=$ SOME

$4=$ NONE

17. How good a worker do you think you are?

$1=$ EXCELLENT

$2=\mathrm{GOOD}$

3.= FAIR

$4=$ POOR

18. How do you feel about most of the people you work with?

$1=$ LIKE THEM VERY MUCH

2 = LIKE THEM A LITTLE

$3=$ DISLIKE THEM A LITTLE

$4=$ DISLIKE THEM VERY MUCH

19. Does your schedule interfere with your sexual activities?

$1=$ RARELY

$2=$ SOMETIMES

$3=$ OFTEN

$4=$ MOST OF THE TIME 


\section{APPENDIX E}

CASE REPORTS FOR

INDIVIDUAL NIGHT WORKERS 


\section{SUBJECT N1:}

Subject $\mathrm{N} 1$ is a 34 year old female who has worked a regular graveyard shift (Sunday through Thursday nights) for five years as a laboratory assistant at a local hospital.

Sleep \& Melatonin Production:

This subject generally sleeps from about 10:00 a.m. until 3:00 p.m. and then naps from about 7:40 p.m until 10:00 p.m. Her dim light melatonin onset is at about 10:30 a.m, producing less than a one hour interval between the average onset of sleep and the onset of melatonin. Nearly all of her sleep (including naps) falls during hours of melatonin production. Her melatonin offset is at about 11:30 p.m. leaving no overlap of work and melatonin production.

On days off she reverts to a fairly normal sleep schedule, although she averages about an extra half hour of total sleep time on days off, sleeping from about midnight until eight or nine in the moming. She does not nap on her days off. Overall, she rates her sleep as fair.

This subject reports frequently feeling tired or sleepy upon awakening, at work and following work. She has occasional problems with insomnia. She never uses sleep medication and uses alcohol as a soporific infrequently.

\section{Psychological Testing:}

This subject scored in the intermediate range on diumal type and as moderately introverted. She scored in the below average range on all of the POMS scales including vigor*. She scored below average on the neurosis scale. (8th percentile). 


\section{General Health and Adjustment:}

Other than hiatal hernia, this subject has no history of chronic or severe health problems. She uses alcohol only occasionally and does not use tobacco products. She rates her health as fair over all.

Frequently Reported Symptoms: Hay fever or sinus trouble, constipation, tight feeling in stomach, bloated or full feeling..

Occasionally Reported Symptoms: Symptoms related to colds or flu, skin rash, back and extremity pain, tinitus, periods of severe fatigue or exhaustion, indigestion, diarrhea, gas, blood in bowel movement, pressure in neck, blurred vision, stomach pains, belching.

Satisfaction With Work and Life: This subject's preferred work schedule is day shift She is moderately dissatisfied with her current night work schedule. She is slightly dissatisfied with the time her schedule allows her to watch tv at home and moderately dissatisfied with her inability to spend enough time with friends and relatives on days off. She noted that her schedule often interferes with sexual activity.

She is moderately dissatisfied with the type of work she does, but feels she is an excellent worker and likes the people with whom she works.

Likes Mest About Schedule: As opposed to other shifts it is relaxing and easy going. I don't have to try to "look busy" if it's slow. I can read or study if my work gets done and I have spare time. I work on my own most of the time. I 
can get most of my school work and writing done while I am at work - saving a lot of my own spare time.

Likes Least About Schedule: The screwed up sleep schedule leaves me tired a lot of the time. Before working graveyard, I was exercising regularly for the first time in my life and had a lot more energy. On this shift I just don't feel like getting started. I try to sleep semi-normally on weekends to see friends and family and end up starting the week low on sleep usually. 


\section{SUBJECT N2:}

Subject N2 is a 39 year old, married female with two grown children who has worked a regular graveyard shift (40 hours per week) for four years and seven months as a night charge nurse at a local hospital.

\section{Sleep \& Melatonin Production:}

This subject appears to have fairly good synchronization among melatonin production, sleep times and work time. She generally sleeps from about 9:40 a.m. until 4:45 p.m. and does not nap. Her dim light melatonin onset is at about 11:15 a.m., producing about a one and a half hour interval between the onset of melatonin production and average sleep time. A full $90 \%$ of this subject's sleep time falls within the hours of melatonin production. This subject's melatonin offset occurs at about 11:30 p.m. just prior to starting the work shift leaving no overlap of melatonin secretion and work.

On days off she reverts to a somewhat conventional sleep schedule, although she tends to initiate sleep rather early, and appears to make-up sleep on days off. She sleeps an average of nearly 13 hours on days off compared to her usual 7 hours on work days. Sleep onset on days off varies between early afternoon and early evening, with wake up times occurring early a.m.

Overall, she rates her sleep as fair. She reports no trouble with insomnia, occasionally wakes feeling tired, rarely feels sleepy at work, but almost always feels sleepy after work. She never takes sleep medication nor does she use alcohol as a soporific. 
This subject scored in the intermediate range on diurnal type and as very extraverted. This subject scored above average on the tension*, anger* and fatigue* scales of the POMS. She also scored within the average range on the neurosis scale (66th percentile). ${ }^{*} \mathrm{~T}$-scores $(\mathrm{T}=55, \mathrm{~A}=60, \mathrm{~F}=60)$.

\section{General Health and Adjustment:}

This subject has no history of chronic or severe health problems. She does not use alcohol or tobacco products. She rates her health as excellent over all.

Frequently Reported Symptoms: No frequently reported symptoms.

Qccasionally Reported Symptoms: Symptoms related to colds or flu, hay fever or sinus trouble, back pain and leg cramps.

Satisfaction With Work and Life: This subject's preferred work schedule is afternoon shift and she is moderately dissatisfied with her current night work schedule. She is moderately dissatisfied with the time her schedule allows her to run errands and attend social gatherings and is very dissatisfied with the time her schedule allows her to spend with friends and family. Her husband is also very dissatisfied with her schedule and complains about it frequently. she notes that her schedule often interferes with sexual activity.

She is satisfied with the type of work she does, feels she is an excellent worker and enjoys the people with whom she works.

Likes Most About Schedule: Able to schedule long weekends. More free time for myself during week. 


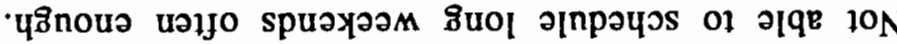




\section{SUBJECT N3:}

Subject N3 is a 42 year old, married male with children who has worked a regular graveyard shift (40 hours per week) for one year and three months as a machine operator at a local plastics firm.

\section{Sleep \& Melatonin Production:}

This subject appears to have fairly good synchronization among melatonin production, average sleep times and work time. He generally sleeps from about 1:45 p.m. until 8:50 p.m. and does not nap. His dim light melatonin onset is at about 3:20 p.m., producing about a one and three quarter hour interval between the average onset of sleep and subsequent melatonin onset. While $85 \%$ of his sleep occurs during the hours of melatonin production, his melatonin offset does not occur until about 3:00 a.m. leaving a three hour overlap between melatonin production and work time.

On days off he reverts to a conventional sleep schedule. Sleeping from about 10:00 p.m. until 6:45 am.

Overall, he rates his sleep as excellent. He reports no trouble with insomnia, rarely feels sleepy at work, occasionally feels sleepy after work but frequently wakes feeling tired. He never takes sleep medication nor does he use alcohol as a soporific. 


\section{Psychological Testing:}

This subject scored in the moderately morning range on diurnal type and as very extraverted. Other than scoring above average on the anger* factor of the POMS, he scored below average on the remaining scales. He scored well below average on the neurosis scale as well (35th percentile).

* $\mathrm{T}$-score $=56$.

\section{General Health and Adjustment:}

Other than nasal/ear surgery in the past year, this subject has no history of chronic or severe health problems. He uses alcohol only occasionally and does not use tobacco products. He rates his health as good over all.

Frequently Reported Symptoms: Skin rashes, severe headaches, hay fever, sinus trouble and gas.

Qccasionally Reported Symptoms: Symptoms related to colds or flu,back pain, tearing or itching eyes, nervous or shaking inside, feeling sweaty or trembly, increased urination, periods of severe fatigue or exhaustion, tight feeling in stomach, hemorrhoids, belching and stomach pains.

Satisfaction With Work and Life: This subject's preferred work schedule is day shift but he reports that both he and his family are moderately satisfied with his current night work schedule. He is moderately dissatisfied with his inability to simply sleep with his wife,although his schedule interferes with sexual activity only sometimes. He is also moderately dissatisfied with the time available for interacting with his children. He is slightly satisfied with the 
type of work he does, feels he is a good worker and likes the people with whom he works.

Likes Most About Schedule: The extra time I can spend with my wife.

Likes Least About Schedule; Not being able to sleep with my wife. 


\section{SUBJECT N4:}

Subject N4 is a 42 year old, single female who has worked a regular graveyard shift (40 hours per week) for two years and eight months as a computer operator in a pathology laboratory at a local hospital.

\section{Sleep \& Melatonin Production:}

This subject appears to have fairly good synchronization among melatonin production, average sleep times and work time. She generally sleeps from about 11:20 a.m. until 4:30 p.m. and naps between 6:00 p.m. and 8:00 p.m. Her dim light melatonin onset is at about 2:48 p.m., producing about a three hour interval between the average onset of sleep and the subsequent onset of melatonin and leaving about half of her sleep time occurring in conjunction with melatonin production. Her melatonin offset occurs just after 11:00 p.m. leaving no overlap of melatonin production and work time.

On days off she reverts to a conventional sleep schedule. Sleeping from about 12:30 a.m. until 8:00 am. She does not nap on days off.

She reports only occasional trouble with insomnia, and occasionally feels sleepy upon awakening or after work but never feels sleepy at work. She never uses sleep medication nor does she use alcohol as a soporific.

\section{Psychological Testing:}

This subject scored in the intermediate range on diumal type and as moderately introverted. She scored below average on all of the POMS scales. She scored above average on the neurosis scale. (84th percentile). General Health and Adjustment: 
This subject has no history of chronic or severe health problems. She does not use alcohol nor does she use tobacco products. She rates her health as excellent over all.

Erequently Reported Symptoms: Persistent cough, belching.

Occasionally Reported Symptoms: Symptoms related to colds or flu, back and extremity pain, tearing or itching eyes, nervous or shaking inside, racing or pounding heart, leg cramps, periods of severe fatigue or exhaustion, indigestion, bloated or full feeling, and difficulty standing for long periods.

Satisfaction With Work and Life: This subject's preferred work schedule is rotating shift but she reports that she is moderately satisfied with her current night work schedule. She is slightly dissatisfied with the time her schedule allows her to attend trainings and professional meetings. Her schedule interferes with sexual activity only sometimes.

She is very satisfied with the type of work she does, feels she is an excellent worker and likes the people with whom she works.

Likes Most About Schedule: Autonomy.

Likes Least About Schedule: Never being able to take a break or lunch.

Having to work at a fast pace all night and having the day shift come in and complain first thing. 


\section{SUBJECT N5:}

Subject N5 is a 29 year old, single female who has worked a regular graveyard shift (40 hours per week) for three years and two months as a medical records clerk at a local hospital.

\section{Sleep \& Melatonin Production:}

This subject appears to have fairly good synchronization among melatonin production, average sleep times and work time. She generally sleeps from about 10:15 a.m. until 8:15 p.m. and does not nap. Her dim light melatonin onset is at about 1:45 p.m., producing about a three hour interval between the onset of melatonin production and average sleep time. Although about $65 \%$ of her sleep occurs in conjunction with melatonin production, her melatonin offset does not occur until nearly 3:00 a.m. leaving a three hour overlap of melatonin production and work time.

On days off she reverts to a semi-conventional sleep schedule. Sleeping from about 8:40 p.m. until 7:30 am. She does not nap on days off.

She reports only occasional trouble with insomnia, occasionally feels sleepy upon awakening, at work and more frequently after work. She never uses sleep medication nor does she use alcohol as a soporific.

\section{Psychological Testing:}

This subject scored in the moderately evening range on diurnal type and as very introverted. She scored slightly below average on the vigor and slightly above average on the anger scales of the POMS. She scored at the average on the neurosis scale. (50th percentile). 
General Health and Adjustment:

This subject reports trouble with gall bladder/liver/pancreas as well as thy roid. She reports a past history of insomnia starting in 1985. She does not use alcohol nor does she use tobacco products. She rates her health as good over all.

Frequently Reported Symptoms: Hay fever, tearing or itching eyes, increased urination, bloated or full feeling, dryness in the mouth and difficulty standing for long periods of time.

Occasionally Reported Symptoms: skin allergies or rashes, feeling sweaty.

Satisfaction With Work and Life: This subject's preferred work schedule is night shift and she reports that she is moderately satisfied with her current night work schedule. The only thing she feels moderately dissatisfied about is the time her schedule allows her to watch t.v. Her schedule rarely interferes with sexual activity.

She is moderately satisfied with the type of work she does, feels she is an excellent worker and generally likes the people with whom she works.

Likes Most About Schedule: I work alone - able to complete my work (other shifts too busy). I have mornings or evenings free to do anything I need or want. 
Likes Least About Schedule: Sometimes I think graveyard is an escape - it's really difficult to meet new people. It's basically a skeleton crew so it's real limited at work. I have to make more effort to meet new people. 


\section{SUBJECT N6:}

Subject N6 is a 22 year old, single female who has worked a semi-regular graveyard shift (three to four on, one to three off) for ten months as an admitting interviewer at a local hospital.

\section{Sleep \& Melatonin Production:}

This subject appears to have fairly good synchronization among melatonin production, average sleep times and work time. She generally sleeps from about 9:20 a.m. until 3:00 p.m. and then naps from about 7:45 p.m until 10:00 p.m. Her dim light melatonin onset is at about noon, producing about a two and three quarter hour interval between the average onset of sleep and the onset of melatonin. About $60 \%$ of her sleep falls during the hours of melatonin production. This subject has a rather long duration of melatonin production and is still secreting melatonin three and three quarter hours after starting her work shift.

On days off she reverts to a fairly normal sleep schedule, sleeping from about 11:20 p.m. until about 9:00 a.m. in the morning. This subject routinely naps during the evening on days away from work from about 6:30 p.m. until 9:30 p.m. Overall, she rates her sleep as fair to good, although she experiences frequent mid-sleep insomnia and reports frequent sleepiness upon awakening, at work and after work. She never takes sleep medication nor does she use alcohol as a soporific. 


\section{Psychological Testing:}

This subject scored in the intermediate range on diumal type and as moderately to very introverted. Other than scoring slightly above average on depression*, she scored well below average on the other POMS scales. She scored at the 92nd percentile on neurosis (the highest in the group).

$\left({ }^{*}\right.$ T-score $\left.=53\right)$.

\section{General Health and Adjustment:}

This subject has past history of asthma and anemia (both appear to be resolved) and a current problem with vertigo for which she is taking Xanax on a daily basis. Other than the aforementioned, this subject has no history of chronic or severe health problems and rates her health as generally good. She does not use tobacco products and drinks alcohol only occasionally.

Erequently Reported Symptoms: Tearing or itching eyes due to allergies, hay fever and sinus trouble.

Occasionally Reported Symptoms: Symptoms related to colds, flu and asthma. Severe headaches, dizziness, periods of severe fatigue and exhaustion, indigestion, gas, nausea or vomiting, bloated or full felling, and stomach pains.

Satisfaction With Work and Life: Although this subject's preferred work schedule is days, she is generally not dissatisfied with the amount of time her schedule allows her to participate in every day activities such as errands and solitary pursuits or the time that it allows her to spend with friends or family. she notes that her schedule sometimes interferes with sexual activity. 
This subject feels that she is an excellent worker and enjoys the people with whom she works. She is very satisfied with the type of work she does and moderately satisfied with her work schedule in general.

Likes Most About Schedule: Allows more flexibility on my time off, better pay, no supervisors.

Likes Least About Schedule: Increase in minor illness (colds, flu) since starting the night shift. since starting nights I have had decreased contact with people (at work and outside of work). Day sleeping interferes with social contacts outside of work. At night there are less staff at hospital, so less personal contact or exchange. 


\section{SUBJECT N7:}

Subject N7 is a 47 year old, single female who has worked a regular graveyard shift (40 hours per week) for seven months as an emergency room unit secretary at a local hospital. In addition this woman works afternoon or swing shift at a second job on a fairly regular basis.

\section{Sleep \& Melatonin Production:}

This subject appears to have little synchronization between melatonin production,average sleep times and work time. Her average sleep onset is at about 9:00 a.m., with sleep offset occurring around 2:15 p.m. She rarely naps in the afternoon. Her melatonin production begins right after she begins work at about 12:30 a.m., producing an interval of about 15.5 hours between her DLMO and average sleep onset. Her entire sleep period falls within the hours of melatonin production but so does nearly all of her work time.

On days off her sleep schedule is somewhat conventional, although she tends to retire rather late, between 1:00 a.m. and 3:00 a.m., awakening at about 7:00 a.m.

She reports frequent insomnia and occasionally feeling sleepy upon awakening and at work and feeling sleepy more frequently after work. She takes sleep medication frequently, but never uses alcohol as a soporific. She reports her sleep as generally fair. 


\section{Psychological Testing:}

This subject scored in the moderate evening range on diurnal type and as moderately extraverted. Surprisingly, she scored in the average range on all of the POMS scales (i.e., below average in tension, depression, anger, fatigue and confusion and above average in vigor). She scored low, at the 30th percentile on the neurosis.scale.

\section{General Health and Adjustment:}

This subject has a past and current history of insomnia and anemia. Other than the aforementioned, this subject has no history of chronic or severe health problems and rates her health as generally excellent. She does not use tobacco products and rarely drinks alcohol.

Frequently Reported Symptoms: Back pain, feeling sweaty.

Qccasionally Reported Symptoms: Stiff/painful extremities, tearing or itching eyes, persistent tingling, nervous or shaking, racing or pounding heart, periods of severe fatigue and exhaustion, indigestion, constipation, bloated or full feeling, trouble digesting food, blurred vision and difficulty standing for long periods.

Satisfaction With Work and Life: This subject's preferred work schedule is days and she reports feeling moderately dissatisfied with her current night shift schedule. She is dissatisfied with the amount of time her schedule allows her to errands. She is celibate. 
This subject feels that she is an excellent worker and enjoys the people with whom she works. She is moderately dissatisfied with the type of work she does.

Likes Most About Schedule: Get away from management politics - close knit group at night. Cooperation and caring for each other.

Likes Least About Schedule: Too little pay and inequities of management. 


\section{SUBJECT N8:}

Subject N8 is a 27 year old, single female who has worked a semi-regular graveyard shift (three to four on, one to three off) for one year and two months as an admitting interviewer at a local hospital.

\section{Sleep \& Melatonin Production:}

This subject appears to have little synchronization among melatonin production, average sleep times and work time. She sleeps on a highly variable schedule, usually attempting to initiate sleep in the morning. At times morning and afternoon sleeps are essentially equal in length, at other times sleep is initiated in the morning and is maintained for a longer period followed by an afternoon nap. Finally, sometimes the preceding relationship is inverted with morning sleep onset resulting in only a short nap and the main sleep occurring in the afternoon or evening. Her dim light melatonin onset is at about 2:45 p.m. On the average about $65 \%$ of her sleep occurs during hours of melatonin production. However, with the high degree of variability in this subjects sleep times, averages probably mean little. This subject had a long duration of melatonin production, with her melatonin offset not occurring until just prior to getting off work at 8:00 a.m.

On days off her sleep schedule is no less variable. She may initiate sleep at 3:00 a.m. or 7:00 p.m. and rarely does she sleep more than five hours at a stretch except occasionally when she has what she describes as a "sleep marathon."

Not surprisingly, she rates her sleep as poor and experiences frequent mid-sleep insomnia. She reports frequent sleepiness upon awakening, at work 
and after work. She never takes sleep medication but uses alcohol as a soporific occasionally.

\section{Psychological Testing:}

This subject scored in the moderate morning range on diumal type and as slightly introverted. Surprisingly, she scored in the average range on all of the POMS scales (i.e., slightly below average on tension, depression, anger, fatigue and confusion and had an average score on vigor). She scored at the 89th percentile for neurosis.

\section{General Health and Adjustment:}

This subject has past history of gastritis. Other than the aforementioned, this subject has no history of chronic or severe health problems and rates her health as generally good. She does not use tobacco products and drinks alcohol only occasionally.

Frequently Reported Symptoms: Hay fever and sinus trouble, indigestion and trouble digesting food.

Occasionally Reported Symptoms: Symptoms related to colds and flu, stiff/swollen/painful muscles, joints, extremities, tearing or itching eyes, tinitus, nervous or shaking, sweaty/trembly, increased urination, leg cramps, periods of severe fatigue and exhaustion, diarrhea, gas, nausea/vomiting, constipation, bloated or full feeling, dry mouth, stomach pains and belching. 
Satisfaction With Work and Life: This subject's preferred work schedule is nights and she reports feeling moderately satisfied with her current night shift schedule. She is dissatisfied with the amount of time her schedule allows her to attend social gatherings and very dissatisfied with the time that it allows her to spend with friends or family. She reports that her schedule almost always interferes with sexual activity. This subject feels that she is an excellent worker and enjoys the people with whom she works. She is moderately satisfied with the type of work she does.

Likes Most About Schedule: Freedom from crowds in public places during the day.

Likes Least About Schedule: Not being able to stay out with friends or have a drink with them. 
SUBJECT N9:

Subject N9 is a 28 year old, married male who has worked a regular graveyard shift (40 hours per week) for one year and three months as a janitor at a local hospital.

\section{Sleep \& Melatonin Production:}

This subject appears to have little synchronization among melatonin production, average sleep times and work time. He generally sleeps from about 9:00 a.m. until 3:00 p.m. and naps from about 7:00 p.m. until 8:15 p.m. His dim light melatonin onset is at about 2:40 p.m., producing about a five hour and 40 minute interval between the onset of melatonin production and average sleep time. Only $6 \%$ of his sleep occurs during hours of melatonin secretion. This subject's melatonin offset occurs at 4:45 a.m., leaving over half of his work time occurring during the hours of melatonin production.

On days off he reverts to a conventional sleep schedule. Sleeping from about 11:00 p.m. until 4:45 am.

Overall, he rates his sleep as fair. He reports frequent trouble with insomnia, and frequently feels sleepy upon awakening, at work and after work. His use of sleep medication was not assessed, but he does not use alcohol as a soporific.

\section{Psychological Testing:}

This subject scored in the intermediate range on diumal type and as very introverted. He scored above average on four of the six POMS scales including depression*, anger*, fatigue* and confusion* and below average on the vigor* scale. He scored high on the neurosis scale as well (72nd percentile). 
(*T-scores: $D=63, A=69, F=54, C=53, V=38$.)

\section{General Health and Adjustment:}

This subject has no history of chronic or severe health problems. He does not use alcohol nor does he use tobacco products. He rates his health as good over all.

Frequently Reported Symptoms: Difficulty with feet and legs when standing for long periods.

Qccasionally Reported Symptoms: Symptoms related to colds or flu, pain or stiffness in extremities, tearing or itching eyes, tinitus, severe headaches, nervous or shaking inside, feeling sweaty or trembly,alarming pain or pressure in chest, indigestion, gas, blood in bowel movement, constipation, blurred vision, and dry mouth.

Satisfaction With Work and Life: This subject's preferred work schedule is day shift but he reports that he is moderately satisfied with his current night work schedule. He reports however, that his family is moderately dissatisfied with his current schedule. He is does not report feeling dissatisfied with the time his schedule allows for activities outside of work and notes that his schedule interferes with sexual activity only sometimes.

He is moderately dissatisfied with the type of work he does, but feels he is a good worker and likes the people with whom he works. 
Likes Most About Schedule: Able to go to church, see my wife, quietness of the work place.

Likes Least About Schedule: My schedule shifts greatly on the weekends and I have a hard time the first part of the week. I can't seem to get refreshed from sleeping. Moods jump around on me. 